\title{
Gradhiva
}

GRADHIV

Revue d'anthropologie et d'histoire des arts

$20 \mid 2014$

Création fiction

\section{L'ivresse et le flacon. Collections ethnographiques et histoire de la culture matérielle du boire en Éthiopie $\left(\mathrm{XVI}^{\mathrm{e}}-\mathrm{xx}^{\mathrm{e}}\right.$ siècle)}

The intoxication and the flask. Ethnographic collections and the history of the material culture of drinking in Ethiopia $\left(16^{\text {th }}-20^{\text {th }}\right.$ centuries)

\section{Thomas Guindeuil}

\section{(2) OpenEdition}

\section{Journals}

Édition électronique

URL : http://journals.openedition.org/gradhiva/2896

DOI : 10.4000/gradhiva.2896

ISSN : 1760-849X

\section{Éditeur}

Musée du quai Branly Jacques Chirac

\section{Édition imprimée}

Date de publication : 1 octobre 2014

Pagination : 242-266

ISBN : 978-2-35744-074-6

ISSN : 0764-8928

\section{Référence électronique}

Thomas Guindeuil, «L'ivresse et le flacon. Collections ethnographiques et histoire de la culture matérielle du boire en Éthiopie (XVI - Xxe siècle) », Gradhiva [En ligne], 20 | 2014, mis en ligne le 01 octobre 2017, consulté le 04 mai 2019. URL : http://journals.openedition.org/gradhiva/2896 ; DOI : 10.4000/gradhiva.2896 



\section{L'ivresse et le flacon.}

Collections ethnographiques et histoire de la culture matérielle du boire en Éthiopie ( $x v l^{e}-x x^{e}$ siècle).

Par Thomas Guindeuil

De nombreux objets, conservés aujourd'hui dans les musées et parfois vieux d'un siècle et demi, permettent d'écrire l'histoire du mobilier domestique en Éthiopie. Ces objets « ethnographiques » devenus objets historiques font rarement partie des matériaux mobilisés par les historiens. À travers l'analyse des corpus de récipients destinés à la consommation d'alcool, berellē et wanç̌a, déposés au musée du quai Branly et à l'Institute of Ethiopian Studies d'Addis-Abeba, cette étude propose de mêler histoire des manières de boire et histoire des collections ethnographiques éthiopiennes. Il pose les bases d'une intégration à la fois plus complète et plus critique des corpus ethnographiques aux sources de l'histoire matérielle et économique de l'Éthiopie. 


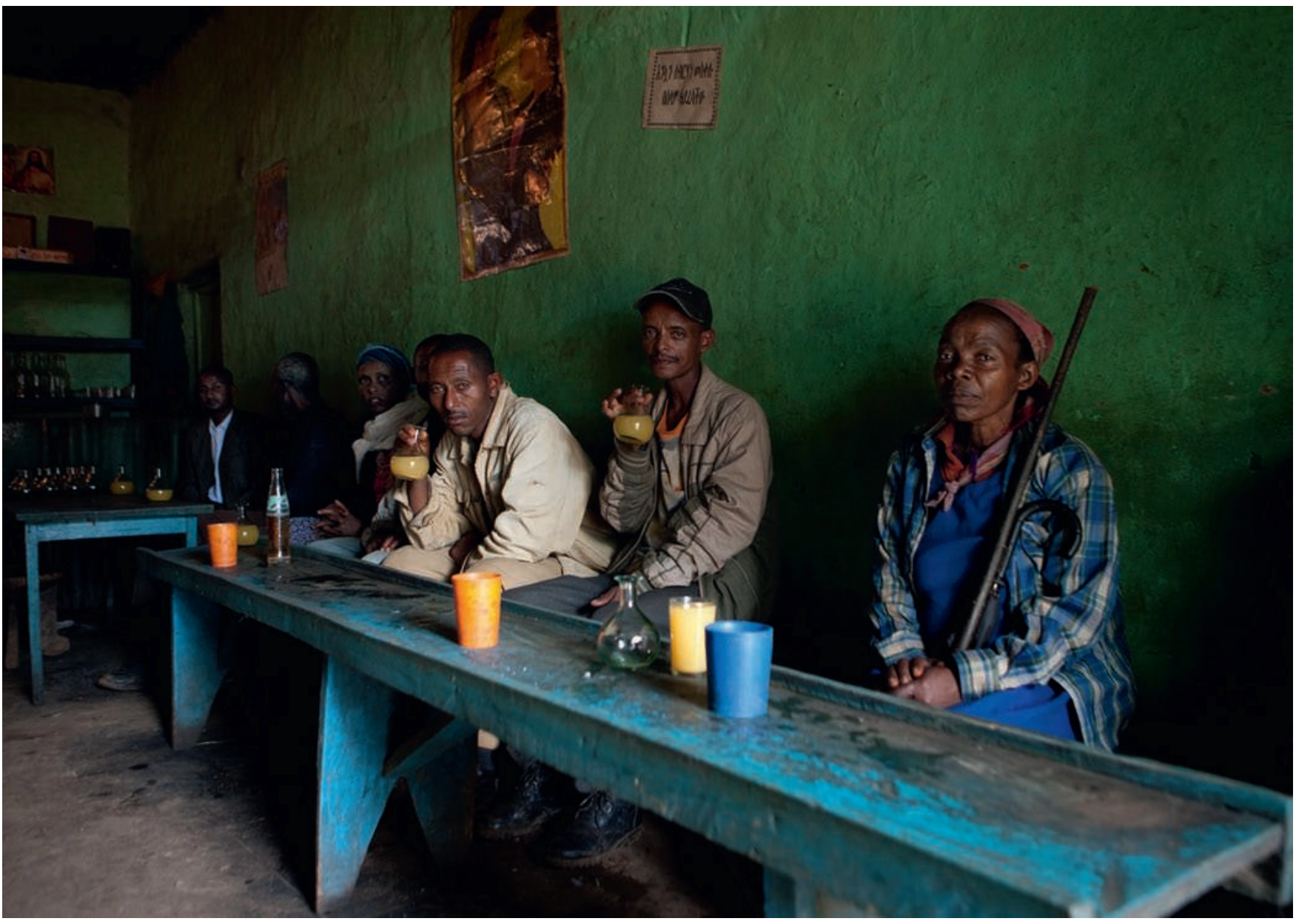

fig. 1

Dans un débit de boisson ethiopien, buveurs d'hydromel utilisant des berellē, 2010 Photo by Eric Lafforgue/ Gamma-Rapho via Getty Images
Chez les chrétiens d'Éthiopie, les temps forts de la vie sociale - réunions familiales, célébrations religieuses - sont marqués par la consommation de deux boissons fermentées préparées par les femmes: la țälla ${ }^{1}$, une bière brune à base de malt parfumée au gēšo (Rhamnus prinoides) ou au șaddo ( $R$. staddo), espèces de nerpruns cultivés à cet effet, et le țäğğ, un hydromel qui partage avec la boisson précédente l'un ou l'autre de ces aromates ${ }^{2}$. À la maison, la țälla est la boisson la plus fréquente, le coût du miel étant généralement prohibitif (Dubois 1989; Guindeuil 2010: 290-293). Tälla et țäğğ peuvent l'un et l'autre être distillés afin d'obtenir de l'eau-de-vie, araqē (aussi appelée 'aräqi ou katikala). À la différence de la bière et de l'hydromel, qui peuvent être élaborés par des particuliers, l'eau-de-vie est exclusivement produite par des bouilleuses de cru qui en font leur profession. Elle fait également partie des boissons festives habituelles, mais elle est progressivement remplacée par des alcools forts de fabrication industrielle.

La consommation de bière et d'hydromel est indissociable de l'usage de récipients spécialisés. Réservé à la consommation de la bière, le wança - également désigné sous le terme d'origine arabe kubbayya ${ }^{3}$ - est le récipient le plus fréquent. II s'agit d'ordinaire d'un gobelet tronconique à fond plat, fabriqué en métal émaillé, présentant des lignes bleues ou noires en haut et en bas, et souvent frappé d'un lion de Judah, symbole de la monarchie éthiopienne abolie en 1974 (fig. 2). La bière, pour sa part, est exclusivement servie dans des berellē, de petits carafons à long col en verre (fig. 1 et fig. 5). Transmis plutôt que vendus, partie intégrante du mobilier domestique de 
tout foyer éthiopien chrétien où ils sont généralement placés pour être vus et conservés amoureusement, ces objets vieux parfois de plus d'un siècle matérialisent le «boire éthiopien». Ils ne sont concurrencés par une vaisselle de verres standards, souvent fabriqués en Chine, que depuis quelques années. Cependant, les wança métalliques et les berellē en verre sont euxmêmes issus de savoir-faire étrangers, en l'occurrence européens, mobilisés de la fin du XIX siècle au milieu du $x^{e}{ }^{e}$ siècle pour conquérir ce marché.

Berellē et wança relient l'histoire des pratiques éthiopiennes du boire à l'histoire des échanges et des voyages. Cet article se propose de revenir sur cet aspect de la culture matérielle, en mobilisant notamment un corpus de sources souvent négligé par les historiens: les collections ethnographiques. En effet, les récipients destinés à la consommation d'alcool peuplent les collections d'objets éthiopiens, tant en France qu'en Éthiopie. Le wança, surtout, est l'un des éléments distinctifs de ces collections. En plus des sources écrites et picturales qui permettent d'aborder la question du boire en Éthiopie chrétienne dans une relative longue durée, nous avons donc choisi d'étudier des objets conservés au musée du quai Branly, à Paris, et au musée de l'Institut des études éthiopiennes (Institute of Ethiopian Studies), à Addis-Abeba 4 .

\section{La collection ethnographique comme témoignage historique}

La collection éthiopienne du musée du quai Branly (MQB), qui s'est enrichie jusqu'au tournant $d u x x^{e}$ siècle, compte 45 récipients à alcool 5 pour la plupart issus de différentes collections anciennes: celles du musée d'Ethnographie du Trocadéro, du musée des Antiquités nationales de SaintGermain-en-Laye et du musée de l'Homme, qui a lui-même hérité des collections du Trocadéro. L'essentiel de la documentation muséale sur les objets éthiopiens du MQB à pour origine les fiches élaborées au musée de I'Homme qui reprennent elles-mêmes souvent les informations recueillies précédemment. Le corpus d'objets qui nous intéresse s'est constitué en plusieurs étapes, qui témoignent de la construction d'une ethnographie de l'Éthiopie en France:

1878: création du musée d'Ethnographie du Trocadéro et organisation de l'Exposition universelle de Paris ${ }^{6}$.

1885: donation de Paul Soleillet, voyageur et marchand présent en Éthiopie entre octobre 1882 et octobre $1883^{7}$.

1890: donation de Jules Borelli, voyageur en Éthiopie mandaté par le ministère de l'Instruction publique, présent en Éthiopie entre septembre 1885 et novembre $1888^{8}$.

1903: donation de la mission dirigée par Robert du Bourg de Bozas 9 (1901-1903).

1930: donation du musée de Saint-Germain-en-Laye ${ }^{\mathbf{1 0}}$ (objets potentiellement conservés depuis la création du musée entre 1862 et 1867).
1. Les termes éthiopiens sont donnés en amharique. La norme de translittération choisie est inspirée de l'Encyclopaedia Aethiopica. Les "e " correspondent à des voyelles de sixième ordre.

2. Sur la commensalité au sein des associations religieuses et les commémorations funéraires, voir Ancel 2011 : $\$ 14,24$ et

29-32. Pour une première ethnographie des boissons éthiopiennes, voir Tegegne Yetesha Work 1958 et Eshete Tadesse 1959. Voir également l'article de l'Encyclopaedia Aethiopica consacré aux boissons (Abbink 2005). Jon Abbink (1997) et Abbebe Kifleyesus (2011) ont étudié les dynamiques contemporaines de la consommation d'alcool en Éthiopie et l'évolution du statut social du boire, respectivement dans le Sud-Ouest éthiopien et en Éthiopie centrale.

3. Sur cette étymologie arabe, voir Kane 1990, vol. I: 1423

4. Cet article a tiré grand profit de la documentation muséale conservée dans ces deux institutions ainsi que des fonds d'archives "Marcel Griaule " et «mission Dakar-Djibouti », déposés à la bibliothèque Éric de Dampierre (Nanterre).

5. Dont quatre manquants. Voir notes ci-dessous.

6. $N^{\circ}$ d'inventaire au MQB dans: 71.1878 .15 .1 et 2 . L'objet est décrit comme un «don du gouvernement égyptien » réalisé à la faveur de l'Exposition universelle. Toutefois, il ne figure pas dans le Catalogue des objets ethnographiques de l'Exposition selon les pavillons (documentation muséale, musée du quai Branly, cote DA000296/15147).
7. Broc 1988: $302-304$ pour une biographie de Paul Soleillet. Le récit de son voyage en Éthiopie et des lettres échangées alors avec la Société de géographie de Rouen et le ministère de I'Instruction publique ont été publiés en 1886 (Soleillet 1886). Voir les objets $n^{\text {os }}$ d'inv. 71.1885.22.79.1 et 2, 71.1885. 22.67, 71.1885.22.54 et 55,

71.1885.22.59, 71.1885.22.60, 71.1885.22. 68, 71.1885.22.61.

8. Broc 1988: 38. Voir le récit de Jules Borelli (Borelli 1890), et notamment son inventaire des objets collectés. Objets $n^{\text {os }}$ d'inv. 71.1890.28.8, 9, 10, 11, 12, 14 (manquant), 15, 16, $17,18,19$ et 20 , puis 71.1890 . 28.13.1 et 2, 71.1890.28.21, et enfin 71.1890.28.4 et 6 (manquants).

\section{Mission scientifique} du Bourg de Bozas 1906. Robert du Bourg de Bozas n'a pas laissé de journal de bord (il est décédé avant la fin de la mission, en Éthiopie), mais la mission a été l'objet d'une publication collective posthume. Un fonds d'archives appartenant au Dr Brumpt, médecin qui accompagnait la mission, est conservé à l'Institut Pasteur, à Paris. Nous n'avons pas encore cherché à y accéder. Voir les objets nos 71.1903 .33 . 26,33 et 34 .

10. $\mathrm{N}^{\circ}$ d'inv. 71.1930 .54 . 989.2 D.

11. $N^{\circ}$ d'inv. 71.1930 .61 .80 . 


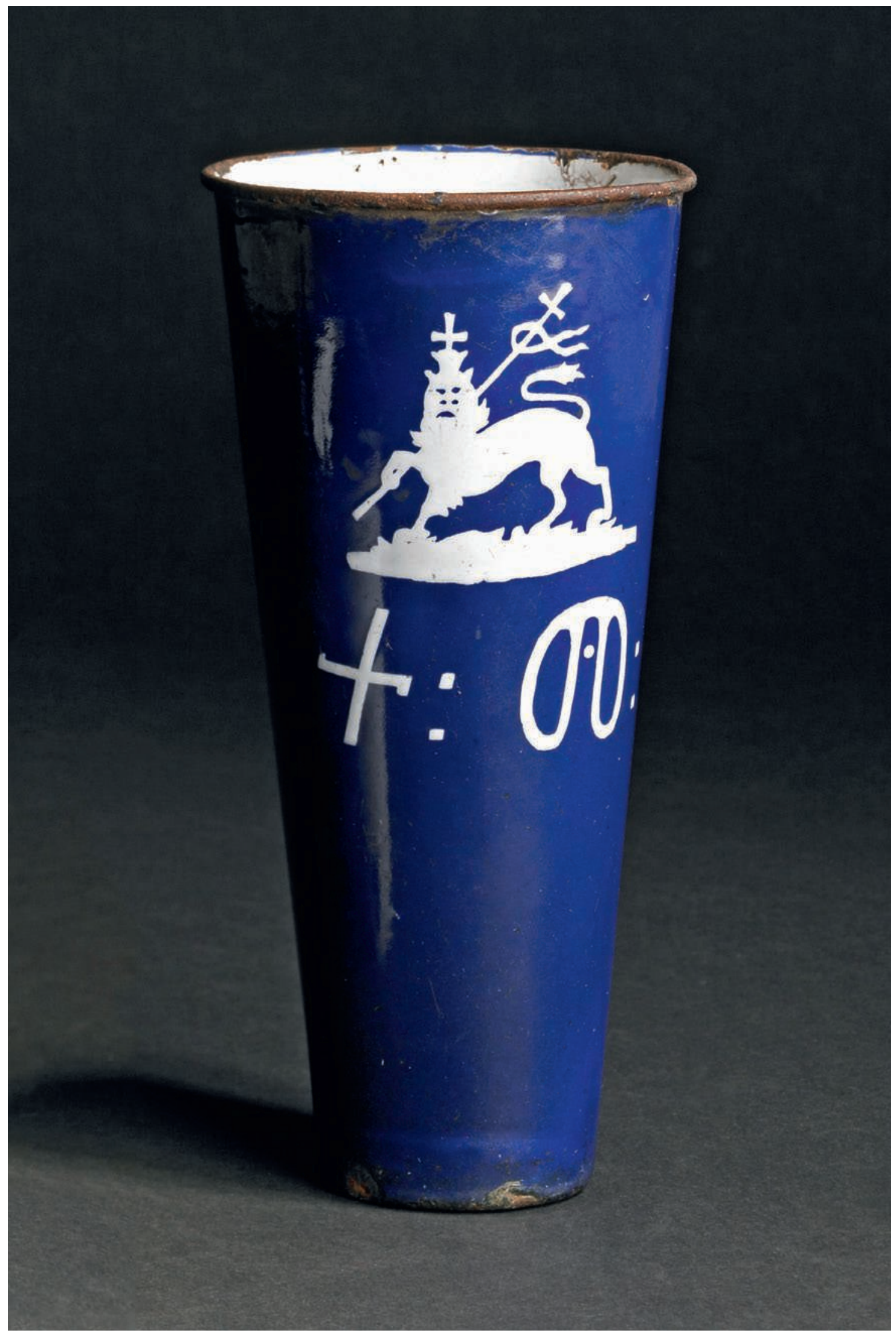


1931: donation de la mission Dakar-Djibouti dirigée par Marcel Griaule - qui entre en réalité sur le territoire éthiopien en 1932. La donation intègre des objets collectés lors de la première mission de l'ethnologue en Éthiopie réalisée en 1928-192912.

1934: donation du linguiste Marcel Cohen, probablement liée à une mission accomplie entre février 1910 et avril $1911^{13}$.

1950: donation du linguiste et ethnologue Joseph Tubiana au musée de l'Homme (créé en 1937). Mission vraisemblablement réalisée entre 1948 et $1950^{14}$.

1961: donation de l'ambassadeur d'Éthiopie en France, Akalē-Wärq Häbtä-Wäld ${ }^{15}$.

1979: donation de M. Levaique ${ }^{16}$.

1999: seconde donation issue de la collection de Marcel Cohen ${ }^{\mathbf{1 7}}$ (décédé en 1974).

La collection éthiopienne du musée du quai Branly permet d'identifier plusieurs catégories de récipients à alcool. Le wança est le type d'objet le plus représenté. Au sein de cette catégorie coexistent plusieurs groupes différenciés par les matériaux utilisés ou leurs particularités formelles. Le wança peut être fabriqué en corne (de bœuf, de buffle ou de rhinocéros, incorporant souvent une pièce en bois à la base), en bois, en céramique ou encore en métal émaillé (fig. 6, 7, 8 et fig. 12). Il peut être équipé d'un étui pour le transport (on parle alors d'un käzäna, fig. 9), ou encore d'un couvercle. La collection du musée du quai Branly comprend également d'autres types de récipients, qui sont incorporés dans les collections françaises dans l'ordre suivant (fig. 3).

Le musée de l'Institut des études éthiopiennes présente une collection de récipients à alcool assez complémentaire de celle du musée du quai Branly mais dont l'histoire est plus ancienne. Ses fiches d'inventaire permettent de recenser au moins 234 objets du boire réunis entre la fin des années 1950 et les années $2000^{18}$. Jusqu'en 1959, les récipients collectés semblent correspondre pour l'essentiel à des dons de particuliers, liés aux activités de recherche de la société d'ethnologie du collège universitaire d'AddisAbeba et aux campagnes du conservateur Stanisław Chojnacki (Pankhurst [A.] 2002). La collection s'enrichit en 1959-1960 d'un grand nombre de céramiques produites par les nonnes et les moines du monastère de Sambo Tree, à Aheya Fäğğ, au sud du Wällo, un lieu réputé pour son savoir-faire en la matière ${ }^{19}$. Le don comporte notamment quatre wança et un berellē en terre cuite ${ }^{20}$. Le registre des acquisitions du musée permet de relever d'autres dons notables, comme celui d'une collection de gobelets émaillés (7 identifiés) offerts à I'Institut des études éthiopiennes par Ato Bä'mnät Gäbrä Amlak le 12 mars 1963. La collection comprend notamment des gobelets en émail «commandés en Allemagne par Sarkis Terzian», un marchand arménien qui fut également un proche conseiller de Menilek $\|^{\mathbf{2 1}}$. Ce don comprend des gobelets portant le sceau de Menilek II, du ras Mäkwännen de
12. La mission de 1928-1929, accomplie avec Marcel Larget, est décrite dans Les Flambeurs d'hommes, publié en 1934 (Griaule 1991 [1934]).

Les objets $n^{\text {os }} 71.1931 .74 .3452$ et 3453 , tous deux des dons du ras Haylu du Goğğam, ont vraisemblablement été rapportés à cette occasion. La mission Dakar-Djibouti, qui franchit la frontière éthiopienne le 20 avril 1932 (Leiris 1996 [1934] : 423), rapporte quatre wanča (nos d'inv. 71.1931.74.3535, 3139, 3452 et 3453).

13. Cohen 1912. II mentionne ses collectes d'objets p. 55. Objet $n^{\circ} 71.1934 .98 .1$.

14. Voir la notice biographique dans les mélanges dirigés par Rouaud (1999: 288). Nº d'inv. 71.1950.99.63.

15. Nos d'inv. 71.1961.86.13, $14,15,16,17$ et 18 .

16. $\mathrm{N}^{\circ}$ d'inv. 71.1979 .28 .19

17. $N^{\text {os }}$ d'inv. 71.1999.13.29 et 31 (ce dernier est manquant). Cette donation a fait l'objet d'un fichier descriptif à part, dont la copie numérique est accessible dans la documentation associée à ces objets.

\section{ci-contre}

fig. 2

Gobelet wança en métal émaillé appelé aussi kubbayya (1916-1930). Cette pièce d'origine française porte un lion de Judah et les initiales du Ras Täfäri Mäkwännen, alors régent de la Couronne. British Museum, Department Africa, Oceania \& the Americas $n^{\circ} 2008,2013.1$. (C) The Trustees of the British Museum. All rights reserved.
18. Ces informations proviennent de la consultation du fichier et du livre des acquisitions du musée de l'Institut des études éthiopiennes, auxquels j'ai eu accès avec l'autorisation du Dr Ahmed Hassen, directeur de l'institut, et du conservateur Ahmed Zaqarya, que je remercie pour leur aide et leurs éclaircissements. Les fichiers sont souvent incomplets, et 1957 est la première date certaine à retenir pour l'entrée dans les collections d'un objet du type étudié. Les nombres d'objets sont donnés ici de façon indicative, la documentation consultée n'étant pas exhaustive.

19. Hecht 1969: 8 et chapitre consacré à l'artisanat du Wällo.

20. Objets nos 1087,1093 , 1521, 1522. Le berellē porte le $n^{\circ} 1033$.

21. Objets nos 1840 à 1852. 


\section{fig. 3}

Répartition par type des récipients à alcool éthiopiens acquis au sein des collections françaises depuis 1878.

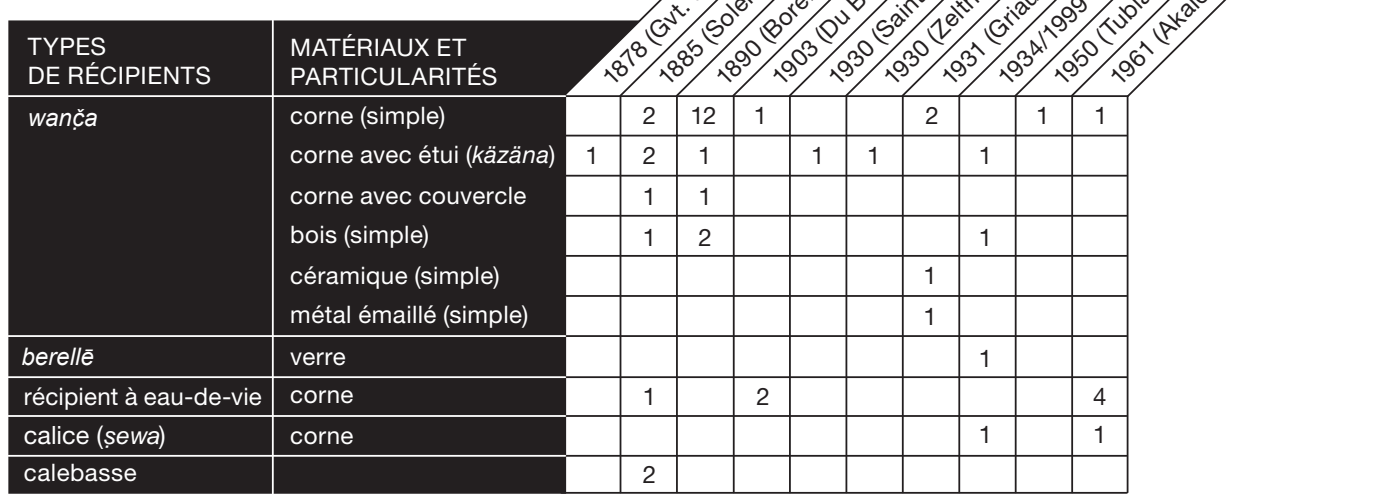

22. Ras correspond au plus haut titre aristocratique éthiopien. Au xx siècle, il est porté par les gouverneurs régionaux. Le ras Mäkwännen Wäldä Mika'ēì, gouverneur de Harar, est le père du ras Täfäri Mäkwännen - futur roi des rois Haylä Śllasē (règne 1930-1974). Sur Sarkis Terzian et son activité d'importateur, voir Adjemian 2011, vol. I: 232 270 et Pankhurst (R.) 2003.

23. L'inventaire réalisé en juin-juillet 2013 n'a pas permis de trouver une documentation relative à des berellē en verre. Ce manque est à mettre au compte de l'absence ou de l'égarement de certaines fiches car quelques-uns de ces berellē très travaillés sont exposés dans les appartements de l'empereur qui font partie des galeries du musée.

24. Dans les réserves, cet équilibre est même renversé car nombre d'objets en corne sont manquants. Un inventaire des collections du musée, comparant les fichiers aux objets conservés, est prévu pour les prochains mois.
Harar22 ou encore les initiales $H: 90$, pour Zäwditu Menilek - fille de Menilek II et «reine des reines» de 1916 à 1930. Les années 1960 sont ensuite marquées par les dons de grandes institutions publiques éthiopiennes comme le ministère de l'Agriculture, en 1964 et 1967, ou le ministère de la Santé publique, en 1970. Après la révolution de 1974, qui renverse la monarchie, l'Autorité des logements et des locations publiques - qui gère la nationalisation des logements privés - donne de nombreux objets au musée, et celui-ci bénéficie, avec la nationalisation des biens du palais impérial, du reversement d'une partie du petit mobilier de l'ancienne maison du «roi des rois» en 1977 et 1978. Beaucoup de donations de récipients restent néanmoins anonymes et, surtout, peu liées à une activité de collecte ethnographique proprement dite une fois passées les années 1950-1960.

La distribution des matériaux et des formes est différente de celle que l'on observe dans les collections du musée du quai Branly. La collection éthiopienne est dominée par la forme wança/kubbayya, mais les berellē y sont des objets communs ${ }^{\mathbf{2 3}}$. Les käzäna et autres wança à couvercle en sont absents. Si la collection est dominée par l'artisanat de la corne (74 objets au moins), ce matériau est talonné par la céramique ${ }^{24}$ (67). À noter, la présence de nombreux récipients en bois et autres matériaux d'origine végétale (35) dont l'usage est souvent difficile à déterminer. On relève la présence de deux à trois récipients en ivoire. Les objets en métal, importés ou produits d'une technologie importée, présentent par leur nombre (46) le décalage le plus saisissant vis-à-vis des collections constituées par les institutions françaises. Décalage d'autant plus significatif que les wança métalliques du musée de l'Institut des études éthiopiennes appartiennent à une période allant du début du $x x^{e}$ siècle à l'occupation italienne de l'Éthiopie (1936-1941), contemporaine d'une partie des collectes européennes. La rareté des berellē en verre et des wança métalliques dans la collection française témoigne manifestement du manque d'intérêt des ethnologues de la première moitié du $x x^{e}$ siècle pour ces biens manufacturés importés, qui sont pourtant tout à fait banals et bien diffusés au moment de leur passage en Éthiopie.

En 1975, une exposition intitulée Éthiopie d'aujourd'hui. La terre et les hommes est organisée au musée de l'Homme: huit gobelets en corne 
de formes diverses, wanç̌a et fenğal (tasse à eau-de-vie) y sont exposés, tous provenant des collections Soleillet, Borelli, du Bourg de Bozas, et de la mission Dakar-Djibouti (Éthiopie d'aujourd'hui... 1975: 94-95). De nos jours, la galerie d'anthropologie du musée de l'Institut des études éthiopiennes ne comporte pas un seul wança en corne, mais présente au contraire une précieuse collection de gobelets et d'autres objets importés fabriqués en métal émaillé à l'effigie des souverains éthiopiens de la fin du XIX et du début du xx siècle. Elle mettait néanmoins l'artisanat de la corne en avant en 1989 - date de publication d'un guide-catalogue - à travers une série de wança collectés dans le Šäwa (Saurat, Ahmad Zekaria et Aklilu Yilma 1989: 9). La dimension ethnographique de ces expositions, largement justifiée par des usages récents ou actuels, fait passer le visiteur à côté du caractère historique d'objets que les Éthiopiens eux-mêmes n'hésitent pas à considérer comme des «antiquités» que l'on collectionne. Quand un Éthiopien - a fortiori un Éthiopien habäša, c'est-à-dire un chrétien des hauts plateaux, communauté politiquement dominante et historiquement liée au pouvoir central - boit dans un wança à l'effigie du lion de Judah, il affirme son lien à un passé révolu: celui des grands banquets publics organisés par Menilek II, ses prédécesseurs ou encore Haylä Śellasē (règne 1930-1974), et où le prestige social était associé au meilleur service et à la mise à disposition des plus beaux récipients.

\section{Une longue quête aristocratique de raffinement et d'exotisme}

Les corpus hétéroclites des collections françaises et éthiopiennes ont été constitués durant une période riche en innovations, qui ne sont que les dernières d'une longue histoire d'introductions et de banalisations de nouveautés dans les pratiques du boire et le mobilier des Éthiopiens. La consommation de țälla comme de țäğğ en Éthiopie chrétienne renvoie à des réalités très anciennes. Le premier texte éthiopien à détailler l'organisation du banquet royal, le Śer'atä geber («Règlement du banquet»), dont la genèse pourrait remonter au $x^{e}$ siècle, mentionne ces deux boissons dans les approvisionnements de la table (Kropp [éd.] 1988: 78). Les premières descriptions européennes des hauts plateaux éthiopiens insistent lourdement sur ce qui est alors perçu comme un remplacement du vin par «de l'eau mélangée d'orge ${ }^{\mathbf{2 5}}$ » et par de l'hydromel. Le chapelain de l'ambassade portugaise envoyée au royaume chrétien d'Éthiopie en 1520, Francisco Álvares, évoque ainsi une «boisson faite d'orge » ou d'autres grains (Álvares 1961, vol. II: 335, 390-391), et un «vin de miel» (ibid., vol. I: 190). À partir du xvII siècle au moins, les élites sociales du royaume chrétien d'Éthiopie développent des pratiques du boire différentes de celles du peuple. La bière est rarement mentionnée à la table des souverains, et l'hydromel se voit concurrencé par des boissons vraisemblablement onéreuses et importées. L'aristocratie éthiopienne emprunte en effet de nouvelles pratiques à des mondes proches, au monde méditerranéen chrétien en particulier.

Le Français Charles Poncet, qui visite Gondär - alors capitale du royaume - entre 1699 et 1702, relève la consommation d'eau-de-vie à la cour du roi lyasu ler (règne 1682-1706) [Poncet 2010: 111-115]. Durant le règne de son petit-fils lyo'as (1755-1769), la reine Mentewwab - qui agit en régente - effectue une donation au clergé de l'église de Däbrä Șäḥay
25. Citation extraite d'une description de l'Éthiopie établie en partie sur renseignements éthiopiens par Poggio Bracciolini, à Florence, dans les années 1430-1440 (Ménard [trad.] $2004: 124)$. 
26. La vigne (Vitis vitifera) fait partie de la flore éthiopienne depuis la période aksumite ( (er-VIII $^{\mathrm{e}}$ siècle) [Boardman 1999] à un moment où, cependant, du vin méditerranéen est vraisemblablement importé dans des amphores par les ports de la mer Rouge (Munro-Hay 1991: 238). Au début du $x v{ }^{\oplus}{ }^{\circledR}$ siècle le jésuite castillan Pero Páez explique avoir tenté de développer la culture de la vigne (Páez 2008: 227) peu de temps avant l'expulsion des missionnaires catholiques au début du règne de Fasilädäs (1632-1667).

27. Voir la description du transport des liquides dans la chronique du règne de Menilek II (Guèbrè Sellassié 1930-1931, vol. I: 230).

28. Abbadie 1881 : col. 936 "coupe, verre, calice».

\section{ci-contre}

fig. 4

Frē Heywat, Comment Menilek II, roi d'Éthiopie, donna un banquet, s.d., détail. Staatliches Museum für Völkerkunde München / State Museum of Ethnology Munich, photo Alexander Laurenzo.
$\mathrm{Q}^{w}$ esq'wam, à Gondär. Celle-ci comprend «du vin [wāyn] et de l'hydromel [mēs] et de la boisson enivrante, à savoir le áräqi [eau-de-vie], en quantité" (Guidi 1910-1912, vol. I [texte]: 186, vol. II [trad.]: 195). La présence de vin à la table des souverains de Gondär est attestée plus tôt dans la chronique du règne de Bäkaffa (1721-1730) [Guidi 1903-1905, vol. III (texte): 295-296, vol. IV (trad.): 316-317], puis dans celle du règne d'lyasu II (1730-1755), où il est écrit que Mentewwab s'abstient, entre autres, de boire du vin et de l'hydromel lorsque son fils, le roi, part en campagne (Guidi 1910-1912, vol. I [texte]: 162, vol. II [trad.]: 172). Le vin, produit à la charge symbolique très forte dans un pays chrétien, est alors vraisemblablement importé ${ }^{26}$. En effet, la viticulture ne s'est développée localement qu'à partir de la première moitié du $x x^{e}$ siècle, d'abord à l'initiative d'étrangers, sans pour autant susciter le développement d'une véritable "culture du vin» (Rouaud 2009: 140). Enfin, la donation de Mentewwab est peut-être l'occasion de la première apparition du terme aräqi, vraisemblablement retranscrit de l'arabe ou d'une autre langue de Méditerranée orientale, dans un texte éthiopien. Le terme amharique áräqi apparaît dans le dictionnaire du missionnaire Charles W. Isenberg, publié en 1841, puis dans celui de l'érudit et voyageur Antoine d'Abbadie en 1881 (Isenberg 1841: 117; Abbadie 1881: col. 481).

La consommation de vin et d'eau-de-vie se développe parallèlement à l'importation en Éthiopie de nouveaux récipients pour la consommation des boissons. Ces innovations illustrent la recherche permanente, de la part de l'aristocratie éthiopienne, d'un boire socialement différencié dans son contenu mais aussi dans son contenant. Néanmoins, il convient de souligner une perma-nence: I'usage de la corne. Au xvi' siècle, Álvares écrit que «pour les grands seigneurs et le prêtre Jean [le roi d'Éthiopie], les cornes de vache sont les verres à vin " (Álvares 1961, vol. I: 98). L'usage de "grandes cornes » est attesté par le jésuite Manoel d'Almeida, au début du XVII siècle, pour le transport des boissons lors du déplacement des armées ou du camp royal (Beckingham et Huntingford 1954: 78). Cette description évoque le qänd (terme qui désigne la corne comme partie de l'animal), grande corne peu travaillée, généralement de buffle, utilisée pour le transport d'importantes quantités de liquide, notamment d'hydromel. Cet objet est encore en usage à la cour de Menilek II et dans les armées à la fin du xix ${ }^{e}$ siècle ${ }^{\mathbf{2 7}}$, et le musée de l'Institut des études éthiopiennes en conserve un grand nombre dans ses réserves. Jusqu'au $x \mathrm{x}^{\mathrm{e}}$ siècle, le travail de la corne ne semble pas devoir être mentionné. Soit qu'il n'existe pas, soit qu'il n'attire pas l'attention des auteurs. Soulignons également l'apparition possible de berellē en céramique dès le $\mathrm{XIV}^{\mathrm{e}}$ siècle dans certaines représentations picturales de scènes religieuses (Campbell 2011: 182-183). Le berellē en verre ne semble s'imposer - dans les textes comme dans les représentations picturales qu'à partir du xIXe siècle.

À la cour de Gondär - capitale du royaume chrétien à partir des années 1630 -, d'autres objets font leur apparition dans les textes. Poncet témoigne ainsi de la consommation d'eau-de-vie dans un «vase en cristal» à la cour d'lyasu ler (Poncet 2010: 111-115). La chronique du règne de Bäkaffa associe consommation du vin et usage de calices, șewa en ge'ez (Guidi 1903-1905, vol. III [texte] : 295-296, vol. IV [trad.] : 316-317) - le terme désigne également le calice dans la liturgie chrétienne ${ }^{\mathbf{2 8}}$. II pourrait s'agir du même type de 


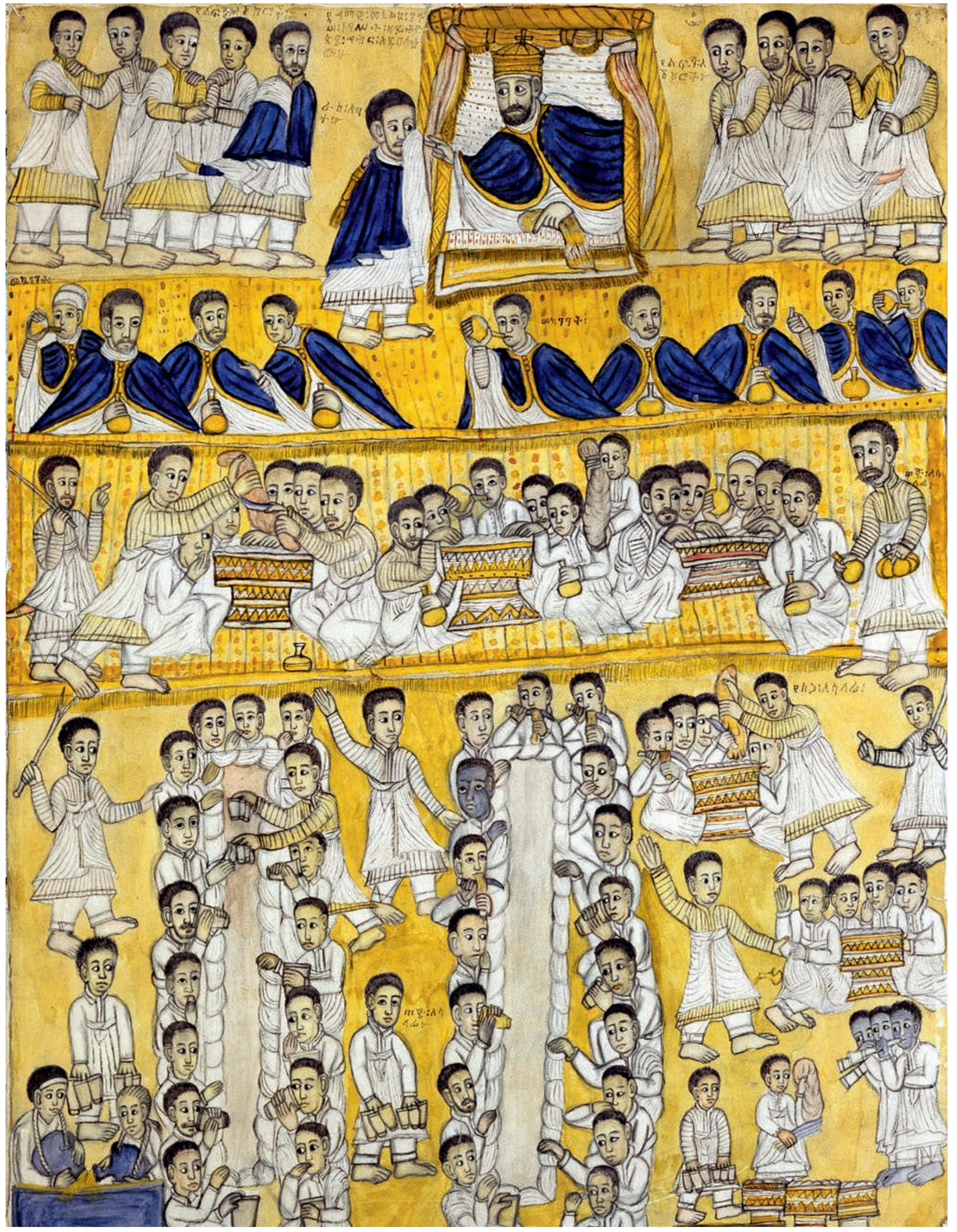




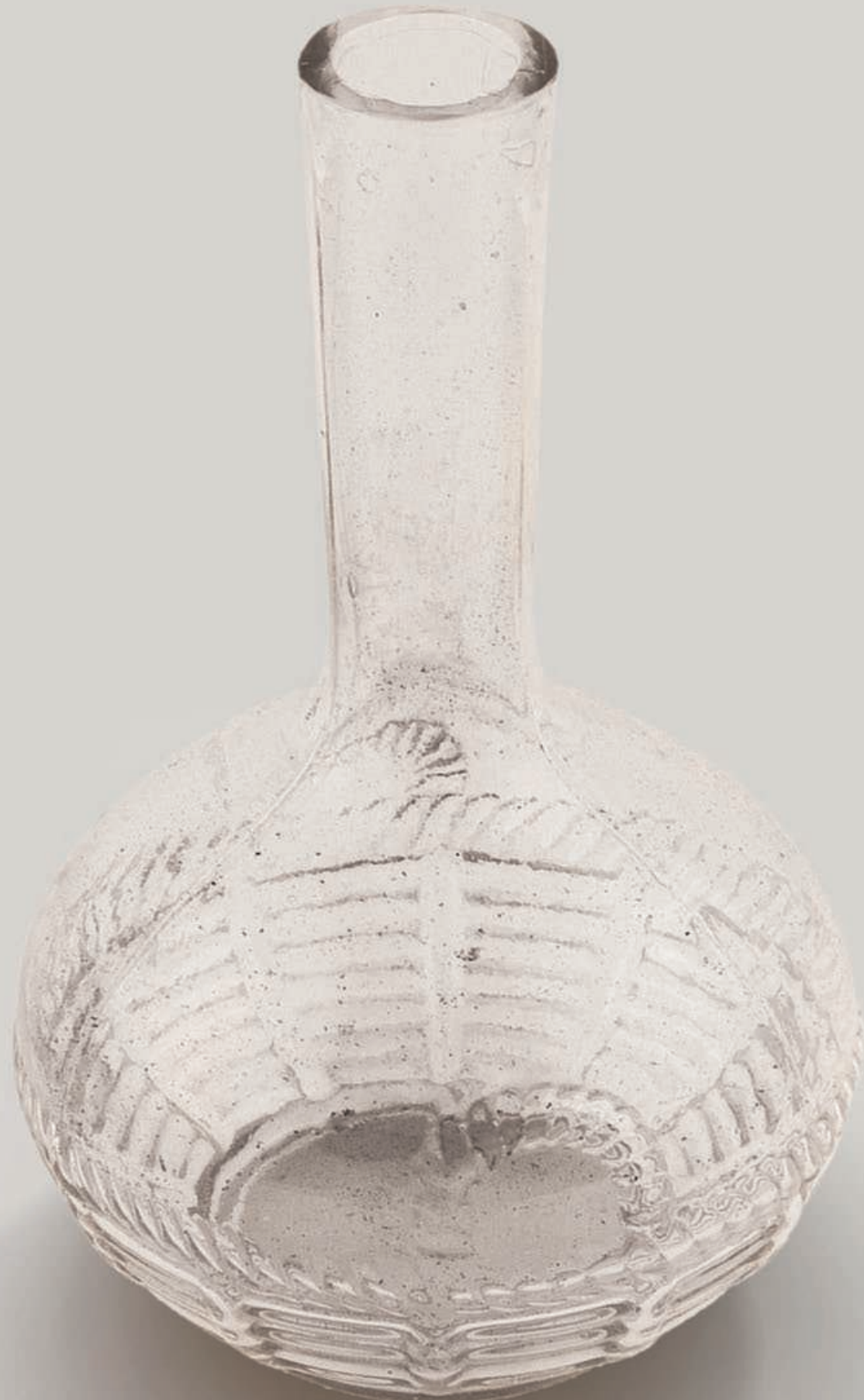


récipient. Le țäğğ est alors servi dans des récipients nommés fenğal (ibid., vol. III [texte] : 296, vol. IV [trad.]: 317), un terme d'origine arabe qui désigne la porcelaine - matériau d'importation - et par extension, en amharique contemporain, les tasses utilisées pour le café29. Les tasses sont également désignées par un mot qui s'impose aujourd'hui en amharique: sini. Ce terme associe cette forme et ce matériau (la porcelaine) à la Chine ${ }^{30}$. Cristal, verres à pied et tasses illustrent la diversité de la vaisselle aristocratique éthiopienne des $x \|^{e}{ }^{e}$ et $x V I I I^{e}$ siècles. Ils constituent des biens de prestige qui marquent le statut d'une élite sociale en demande de distinction, de raffinement et d'exotisme.

\section{Un "boire éthiopien" unifié dans sa forme par le wança}

La fin du $x \mathrm{XIII}^{\mathrm{e}}$ siècle voit l'effondrement du pouvoir effectif des rois

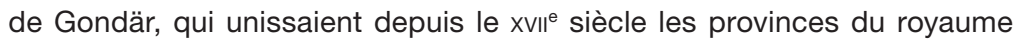
chrétien d'Éthiopie - l'Éthiopie septentrionale et centrale contemporaine ainsi que l'Érythrée. L'Éthiopie chrétienne se morcelle sous la domination de potentats régionaux. L'arrivée sur le trône de Tēwodros II (règne 1855-1868) marque le début de la reconstruction de l'unité de cet ensemble, puis de la construction de l'État éthiopien contemporain, achevée par Menilek II dans la dernière décennie du $x x^{e}$ siècle. Celle-ci confère au territoire des rois chrétiens des dimensions beaucoup plus importantes que celles des seules provinces chrétiennes en incorporant les riches régions méridionales, notamment le Käfa, terminus des routes commerciales séculaires qui traversent les hauts plateaux éthiopiens au départ des ports de la mer Rouge et du golfe d'Aden. Cette période est également marquée par une plus grande ouverture de l'Éthiopie aux affaires internationales. Le Šäwa, région centrale du nouvel État, a été et est encore dans les dernières décennies $\mathrm{du} \mathrm{XIX}^{\mathrm{e}}$ siècle une destination parcourue par des voyageurs européens aux visées très générales - aventure, commerce, science, diplomatie, etc. - dont, parmi les plus tardifs, Soleillet et Borelli. Notre connaissance de la culture matérielle des hauts plateaux éthiopiens et des circuits économiques de la région s'élargit considérablement $\mathrm{au} \mathrm{XIX}^{\mathrm{e}}$ siècle du fait des récits et notes de voyage ainsi que des collectes d'objets, le plus souvent liés à des activités quotidiennes.

Dans ce contexte, le travail de la corne apparaît dans les sources historiques. Le wança est l'un des objets qui attirent l'attention des voyageurs pour leurs qualités esthétiques. II n'est alors question que de wança en corne. «Ces cornes, écrit le britannique Henry Salt en 1809, sont amenées au Tigré depuis Gondar; mais elles sont surtout manufacturées, ou transformées en gobelets, dans le Gojam. » (Valentia 1809, vol. III : 149) Dans son dictionnaire de la langue amharique, Isenberg définit le wança comme une "corne à boire, avec laquelle les Abyssins boivent leur bière et leur eau » (Isenberg 1841: 149); Abbadie évoque plus précisément une «coupe à boire, en forme de corne tronquée, et faite en corne de vache» (Abbadie 1881: col. 675). Ce dernier précise que l'on réserve le terme $k^{w}$ älba aux gobelets fabriqués en corne de buffle (ibid.: col. 675 et 598). Wança et $k^{w}$ älba se différencient donc du qänd, la corne brute, par une opération de transformation de celle-ci, ce que notent les voyageurs Edmond Combes et Maurice Tamisier en 1838 :
29. Le terme amharique dérive de l'arabe, et semble désigner autant un objet que son matériau. Charles W. Isenberg (1841: 195) le traduit par "tasse", "petit verre à boire ", Antoine d'Abbadie (1881: col. 1007) par «tasse en faïence ou en porcelaine. - faïence, porcelaine, coupe».

30. Baeteman (1929: 238) définit sini, ou seni, comme une "petite tasse à café ". Pour l'étymologie, voir Kane 1990, vol. I: 534.

\section{ci-contre}

fig. 5

Carafon en verre berellē (don Marcel Cohen, 1934). Musée du quai Branly n71.1934.98.1. (C) musée du quai Branly. 
31. Sänga est une race bovine réputée pour sa viande et la longueur de ses cornes.

32. Duchesne-Fournet 1909, vol. II : pl. xviii. II s'agit respectivement des objets inventoriés sous les nos d'inv. MQB suivants: 71.1890 .28 .12 ( ${ }^{\circ}$ MET 26944),

71.1890 .28 .18 (26950) et 71.1878.15.1-2 (12482)

33. Bonacci 2011 : 102-103; Imbert-Vier 2011: 168 et n. 120. Cette collection n'a pas été versée à celles du musée d'Ethnographie du Trocadéro.

34. Fonds Marcel Griaule/ Papiers/Grands fichiers/ Fichier Éthiopie 1931-1937. Catégorie «Industrie»; sous-catégorie «Wancha?". Réf. fiche: fr/up10/ mae/lesc/fmg_pap_gf aet3137_086_065_008. Catégorie "Métier»; sous-catégorie "Tour à gobelet [pour le travail de la corne] ". Réf. fiches: fr/ up10/mae/lesc/fmg_pap _gf_aet3137_086_013 (bibliothèque Éric de Dampierre, Nanterre).
Les cornes de divers animaux sont employées à une foule d'usages: on en fait des poignées de sabre et des manches d'outils; celles des bœufs sanga ${ }^{31}$ sont conservées dans toute leur longueur, et on les remplit ordinairement d'hydromel; les cornes de bœufs domestiques sont transformées en écritoires, en salières ou poivrières; celles dont on se sert pour boire ont la forme d'un gobelet: nous avons déjà dit qu'elles étaient d'un travail fini; celles qu'on remarque chez les princes sont d'un noir de jais ou d'une blancheur limpide. (Combes et Tamisier 1838, vol. IV: 83-84)

Le travail de la corne en vue de la création des wança nécessite l'usage d'un tour. Charles Johnston, qui visite le Šäwa dans les années 1840, livre une description relativement précise de ce travail et de l'outillage employé, qui suggère qu'il a lui-même observé cette technique de fabrication (Johnston 1844, vol. II: 332-335). Combes et Tamisier évoquent également l'usage du tour pour le travail de l'ivoire. "Les ouvriers de SahléSélassi [Säḥlä Śellāsē, roi du Šäwa], écrivent-ils, ne ménagent pas leurs dents d'éléphant; ils en font des milliers de bracelets et des pions pour jouer aux échecs." (Combes et Tamisier 1838, vol. IV: 83-84) Les artisans qui fabriquent des wança au tour forment un personnel spécialisé, appelé wança anțač - du verbe anäțäțä, travailler au tour -, les «tourneurs de wança ». L'expression apparaît dans le dictionnaire d'Abbadie (1881: col. 540).

Objet singulier, le wança en corne devient un artefact ethnographique en entrant dans les collections européennes publiques et privées. II se voit consacrer une planche illustrée de l'ouvrage-bilan de la mission scientifique dirigée par Jean Duchesne-Fournet en Éthiopie entre 1901 et 1903, publié en 1909. Y figurent notamment trois objets conservés au musée d'Ethnographie du Trocadéro - deux wança de la collection Borelli et un käzäna entré dans les collections dès 1878 , issu d'un «don du gouvernement égyptien ${ }^{\mathbf{3 2}}$ ». Les autres objets représentés viennent de la collection privée du Dr Vitalien, médecin d'origine antillaise attaché à Menilek II dans les années $1900^{\mathbf{3 3}}$. Exploitant les informations recueillies lors de ses missions en Éthiopie, Griaule consacre un article aux wança et à leur fabrication, publié en 1941 dans le Journal de la Société des africanistes. On y trouve notamment un précieux schéma du tour éthiopien, repris des fiches constituées lors de la mission Dakar-Djibouti ${ }^{34}$. La corne est sectionnée, puis chauffée, distendue, empalée sur une pièce de bois au bout conique de la largeur souhaitée et terminée par un manche plus fin qui sert de mandrin. L'ensemble est fixé sur un axe longitudinal dont la rotation est activée à l'aide d'une courroie enroulée autour du mandrin. L'artisan fait tourner le gobelet dans les deux sens pour imprimer sur la corne des filets réguliers avec une pointe métallique. Le plus souvent, les gobelets conservés dans les musées français et éthiopien comportent deux à quatre filets au niveau de l'ouverture, et un motif similaire à proximité du pied. Certains sont entièrement travaillés (fig. 10). Couvercles, pieds, fonds en corne sont également travaillés au tour, mais la plupart des gobelets sont fermés par un opercule de bois fixé avec des chevilles (Griaule 1941: 201-206).

Le Goğğam, région chrétienne du nord-ouest des hauts plateaux où Salt situe le meilleur de la production, est aussi la région d'enquête privilégiée 
de Griaule. L'idée d'une spécialité artisanale locale est réaffirmée dans le catalogue de l'exposition Éthiopie d'aujourd'hui du musée de l'Homme, publié en 1975 (Éthiopie d'aujourd'hui... : 94), puis reprise dans le guidecatalogue du musée de l'Institut des études éthiopiennes publié en Éthiopie en 1989 (Saurat, Ahmad Zekaria et Aklilu Yilma 1989: 8). Notons que le premier catalogue est préfacé par Chojnacki, alors conservateur du musée de l'Institut des études éthiopiennes. Mais la collecte atteste d'une distribution large de ces objets, qui prend la forme du vaste empire de Menilek II. Borelli note dans son récit la présence de wança en corne de bœuf et de buffle en vente sur le marché de Ğimma, grande place commerciale du SudOuest éthiopien (Borelli 1890: 288). Le wança est manifestement un bien destiné à circuler, qui ne peut être cantonné à une seule origine géographique ou à une seule région d'usage. Il faut ainsi souligner que les régions productrices identifiées - le Goğğam et, dans une moindre mesure, le Šäwa sont des carrefours où se croisent les itinéraires caravaniers qui relient le Sud éthiopien au Nord, abondamment décrits par les voyageurs du XIXe siècle (voir par exemple Beke 1844 et Abbadie 1890). Ce sont aussi des régions "tampons ", situées à la limite des vieux territoires chrétiens et du monde oromo - les Oromo que le Dr Étienne Mérab, médecin qui succède au Dr Vitalien auprès de Menilek II, estime être les spécialistes du travail de la corne et de l'ivoire (Mérab 1929: 401).

Enfin, le wança voyage également du fait des usages qui lui sont assignés. Enserré dans un käzäna, il s'agit d'un ustensile indispensable et caractéristique de la panoplie du voyageur éthiopien (Rouaud 2009: 129). Le wança est aussi décrit comme un outil de mesure utilisé sur les marchés de toute l'Éthiopie. En visite sur le marché de Bonga, dans le Käfa, Soleillet explique que «le café ne se vend pas au poids, mais à la mesure, au ouancha, gobelet en corne, d'une capacité d'environ trois quarts de litre » (Soleillet 1886: 190). L'une des fiches de travail de Griaule fait également du wança l'instrument privilégié des mesures de liquide, et donc de volume ${ }^{35}$. Cet usage est noté sur la fiche du wança métallique collecté en Érythrée par la mission Dakar-Djibouti ${ }^{36}$. Enfin, le wança peut être un bien de prestige que l'on offre à un hôte de marque de passage à la cour. Soleillet mentionne deux wança - que l'on retrouve dans son don au musée - qui lui furent respectivement offerts par les reines de Gera et de Ğimma, deux royaumes oromo du Sud éthiopien ${ }^{37}$. De même, deux wança en corne de rhinocéros ont été donnés par le ras Haylu Täklä Haymanot, gouverneur du Goğğam, à Griaule lors de son passage en 1928-1929, avant d'être versés aux collections ethnographiques ${ }^{38}$. Notés dans les fiches d'inventaire «oromo» dans les premiers cas, puis «amhara» dans les seconds ${ }^{39}$, ces wança «diplomatiques » semblent pourtant exprimer une culture matérielle commune aux élites sociales du centre et du sud de l'Éthiopie. Ces différents usages laissent deviner une grande variabilité dans leurs qualités esthétiques et techniques. La forme tronconique marquée par le travail du tourneur unit néanmoins tout l'empire.

Cette absence d'attache géographique précise entre en contradiction avec les critères de classification des musées ethnographiques. Si dans la fiche d'inventaire du musée de l'Homme consacrée au berellē rapporté par Cohen, il est admis que l'objet, d'origine européenne, est de distribution générale en «Abyssinie ${ }^{40}$ », les wança se voient assigner des origines plus
35. Fonds Marcel Griaule/ Papiers/Grands fichiers/ Fichier Éthiopie 1931-1937. Catégorie «Industrie»; sous-catégorie "Mesures». Réf. fiche: fr/up10/mae/lesc/ fmg_pap_gf_aet3137_086_ 065_006 (bibliothèque Éric de Dampierre, Nanterre).

36. Voir la fiche d'inventaire du musée de l'Homme de l'objet enregistré sous le nº d'inv. MQB 71.1931.74.3139.

37. Soleillet $1886: 172$ et 178 ( $n^{\circ}$ d'inv. MQB

71.1885 .22 .79 .1 et 2

et 71.1885.22.67).

38. $N^{\circ}$ d'inventaire $M Q B$ 71.1931.74.3452 et 3453 .

39. Fonds mission DakarDjibouti, bibliothèque Éric de Dampierre, Nanterre, fiches objets nos 31.74 .3452 et 3 .

40. Voir la fiche d'inventaire dans la base de données TMS du MQB, n 71.1934.98.1. 


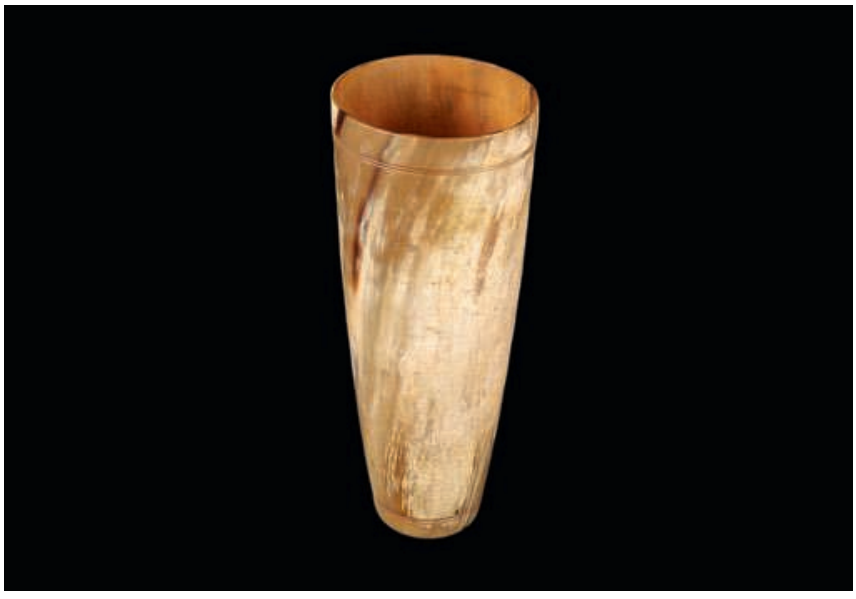

fig. 6

Gobelet wanča simple en corne de bœuf (don Jules Borelli, 1890). Musée du quai Branly nº 71.1890.28.8. @ musée du quai Branly.

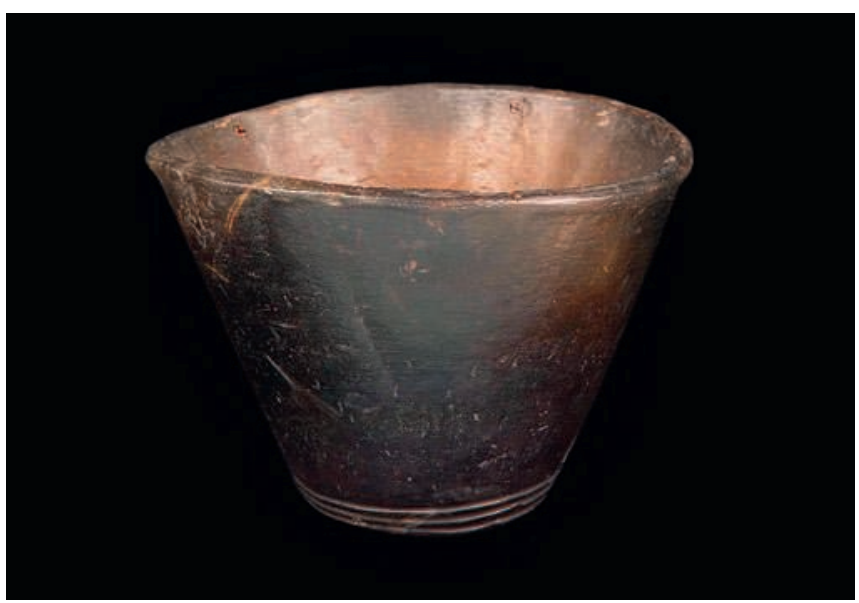

fig. 8

Gobelet wança en terre cuite (don mission Dakar-Djibouti, 1931). Musée du quai Branly. n71.1931.74.3535. @ musée du quai Branly.

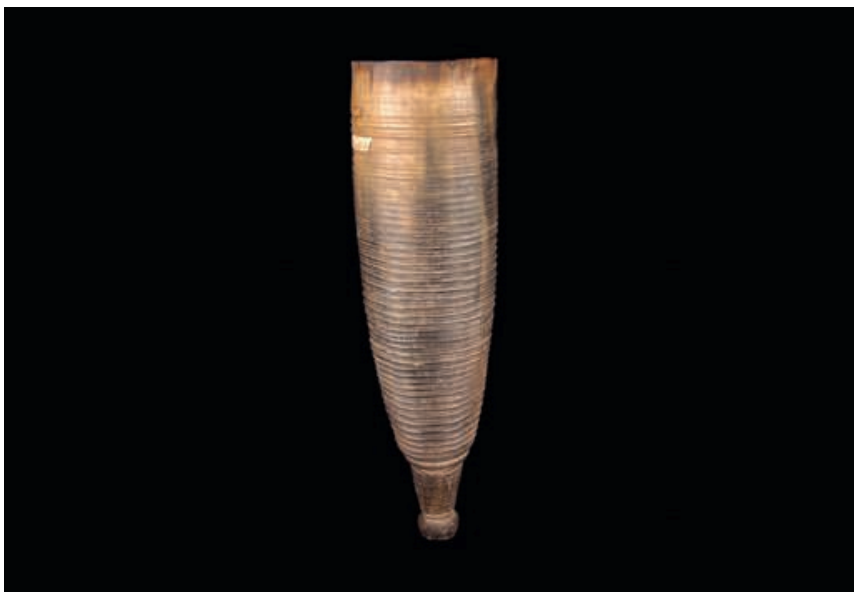

fig. 10

Gobelet wanča en corne de bœuf très travaillé (don Paul Soleillet, 1885).

Musée du quai Branly n71.1885.22.60. (0) musée du quai Branly.

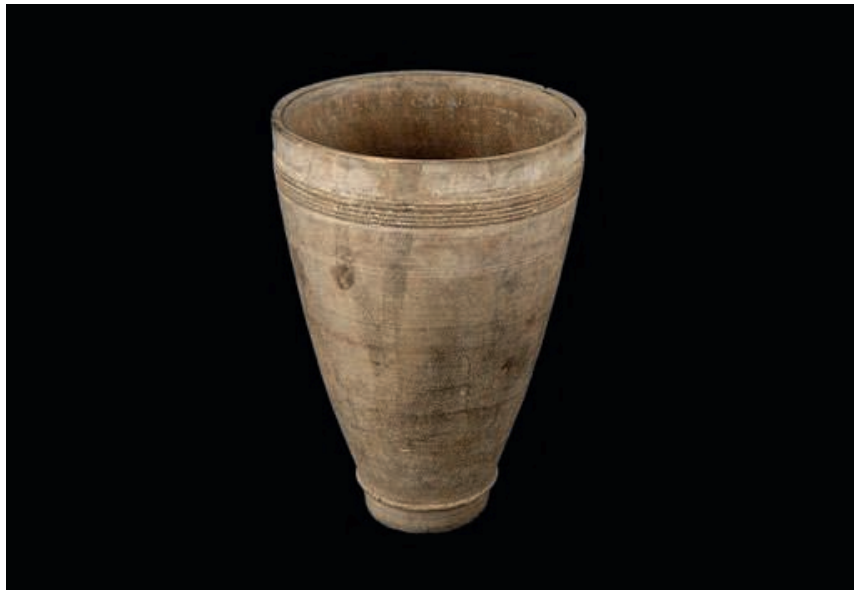

fig. 7

Gobelet wanča en bois (don Marcel Cohen, 1999).

Musée du quai Branly n71.1999.13.30. @ musée du quai Branly.

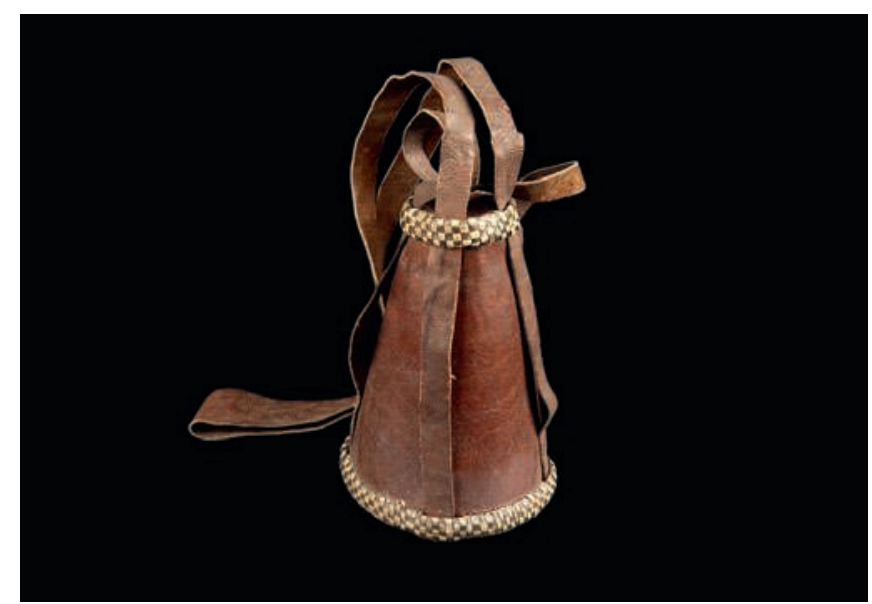

fig. 9

Étui de transport käzäna pour gobelet à boire (don gouvernement d'Égypte, avant 1878). Musée du quai Branly n71.1878.15.1.2. @ musée du quai Branly.

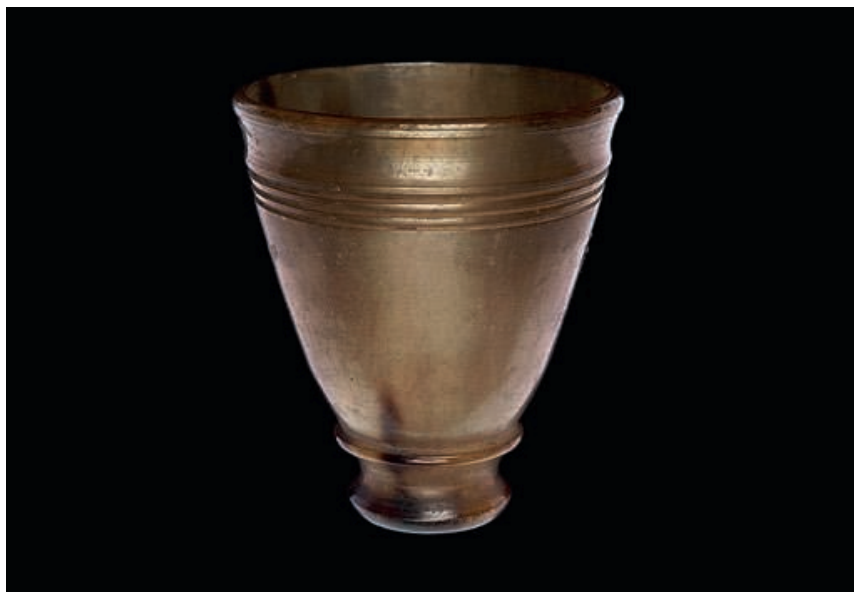

fig. 11

Verre à eau-de-vie mäläkkiya en corne (don Paul Soleillet, 1885).

Musée du quai Branly n71.1885.22.61. @ musée du quai Branly. 
précises: régions du Šäwa, de Ğimma, du Goğğam, de Harar ou Érythrée, ethnonymes «Amhara» ou «Oromo», etc. Pourtant, rares sont les objets que l'on peut resituer avec précision dans leur contexte de collecte. De façon plus anecdotique, il faut également souligner que certains ethnonymes ont été attribués lors des inventaires, au risque de trahir les écrits du collectionneur lui-même. C'est le cas de plusieurs wança de la collection Borelli, dont une liste est dressée dans son ouvrage. Les informations qu'il y donne ne se retrouvent pas systématiquement dans les fiches du musée de l'Homme. Ainsi, un käzäna originaire de Ǧimma devient «Amhara41 " et un wança avec couvercle, très similaire à celui rapporté par Soleillet du royaume oromo de Gera quelques années plus tôt, est étiqueté «Amhara" alors qu'il était initialement dépourvu de toute indication d'origine ${ }^{\mathbf{4 2}}$.

\section{De la corne au métal: la banalisation de l'exogène}

Régulièrement identifiés comme des "bouteilles vénitiennes" par les voyageurs européens (Johnston 1844, vol. II: 170-171; Parkyns 1868: 217-218), les berellē semblent connaître une diffusion foudroyante chez les consommateurs d'hydromel du nord de l'Éthiopie au point de nécessiter, à la fin $d u x I x^{e}$ siècle, la création par les usines européennes de modèles plus luxueux, distincts d'un berellē ordinaire devenu par trop banal et répandu (Campbell 2011: 179-205). Le temps est venu des grandes commandes. Le voyageur français Rochet d'Héricourt, qui visite le royaume du Šäwa dans les années 1840, écrit qu'«à la table royale, on [...] versait [l'hydromel] aux personnes de distinction, dans de petits bocaux en verre nommés birillés, et aux personnes de rang inférieur, dans des gobelets de corne [...]" (Rochet d'Héricourt 1841: 153-156). C'est en effet le palais qui fournit la vaisselle par centaines et milliers de pièces. La chronique du règne de Menilek II mentionne des serviteurs spécialisés: porteurs de berellē et porteurs de wança (Guèbrè Sellassié 1930-1931, vol. I: 224 et 226). Certaines représentations picturales des banquets de Menilek II illustrent ce propos, tel le tableau Comment Menilek II, roi d'Éthiopie, donna un banquet de Frē Heywat (fig. 4) où les berellē sont en verre et les wança manifestement en corne ${ }^{43}$.

Si le wança est a priori un objet moins prestigieux que le berellē, les deux objets sont avant tout distingués par des usages différents: le premier sert à consommer la țälla, boisson moins valorisée que le țäğğ qui lui est bu dans un berellē. L'utilisation du wança est néanmoins plus générale: la bière artisanale est bue dans tous les milieux sociaux et, malgré certaines variations, est consommée par de nombreuses populations de la nouvelle et vaste Éthiopie (voir par exemple Abbink 1997 : 10-11; Abbebe Kifleyesus 2002: 263). La forme prise par le wança est donc également un enjeu de distinction. Dans son article, Griaule en propose certains critères: la qualité du gobelet s'évaluerait à son évasement (on parle alors d'afa galälä ou d'afa saffi - «bouche écartée» ou «bouche large»); les gobelets les plus grands, nommés ankabo, sont «réservés aux soldats ${ }^{44}$ "; la corne de bœuf est le matériau le moins valorisé, contrairement à la corne de rhinocéros ou à l'ivoire ${ }^{45}$. En outre, on peut supposer que la présence d'un couvercle - comme c'est le cas pour l'un des présents royaux offerts à Soleillet - et, plus généralement, la complexité du travail de l'artisan entrent en considération.
41. Borelli 1890: 496. $N^{\circ}$ d'inv. MQB: 71.1890 .28 .21 (260 dans l'inventaire de Borelli).

42. Ibid. $N^{\text {os }}$ d'inv. MQB: 71.1890.28.13.1 et 2 (265 dans l'inventaire de Borelli)

43. Les gobelets représentés sont transparents ou translucides, ce qui exclut au moins le bois et la céramique. On ne connaît pas, dans les collections, de wança fabriqués en verre.

44. Marcel Griaule s'appuie ici sur Guèbrè Sellassié 1930-1931, vol. I : 343. Il compte aussi la planche de la mission DuchesneFournet parmi ses sources. Thomas Leiper Kane (1990, vol. II : 1226) définit le terme par «large horn cup».

45. Griaule 1941 : 206-207. Sur la chasse au rhinocéros et à l'éléphant, voir Mérab 1929: 401. 
46. $N^{\circ}$ d'inv. MQB 71.1931.74.3139.

\section{ci-contre}

fig. 12

Gobelet wança en métal émaillé (kubbayya) à l'effigie de Menilek II (don mission

Dakar-Djibouti, 1931). Musée du quai Branly $n^{\circ} 71.1931 .74 .3139$.

(C) musée du quai Branly.
Probablement plus valorisée que la corne de bœuf, la corne de buffle est très utilisée par l'artisanat en Éthiopie (Salt 1814: 258-259). Depuis l'époque gondarienne, et probablement plus tôt, le buffle est l'objet de chasses aristocratiques qui se déroulent principalement dans les basses terres (Ahmad 1988). Les cornes et la peau du buffle - qui sert à fabriquer des boucliers - apparaissent donc comme des trophées. Borelli signale l'existence de chasseurs "professionnels" qui parcourent de longues distances, du royaume de Gera aux basses terres occidentales (Borelli 1890: 301). La chasse, mais également une épizootie de peste bovine, survenue entre 1888 et 1892 (Pankhurst [R.] 1985: 57 sq.), semblent avoir largement réduit cette ressource au début du $x x^{e}$ siècle, comme le fait remarquer un guide éthiopien à Robert E. Cheesman, consul britannique en Éthiopie de 1925 à 1934 (Cheesman 1936: 327).

Voyageurs et ethnologues s'accordent pour faire de la corne le matériau «normal» du wança. La rareté relative dans la collection française des matériaux moins nobles comme le bois et la terre cuite, très présents dans la collection d'Addis-Abeba, suggère l'existence d'un important biais de collecte. Le tour est pourtant employé pour la conception de wança à partir d'autres matériaux que la corne, plus prestigieux comme l'ivoire, moins onéreux comme le bois. Les wança en bois travaillé au tour sont ainsi présents dans les collections Soleillet et Borelli. L'usage du tour n'existe pas dans l'artisanat de la céramique en Éthiopie. La collection de wança en céramique du musée de l'Institut des études éthiopiennes, acquise durant la seconde moitié du $x x^{e}$ siècle, ainsi que l'unique objet de ce type de la collection du quai Branly témoignent néanmoins du rôle d'inspirateurs joué par les tourneurs: la plupart des objets sont décorés de filets, généralement positionnés comme ceux (tracés au tour) des wança en corne, en haut et en bas du gobelet (fig. 8). Ce sont les artisans (wanç̌a anțaç) et leurs clients qui imposent la norme esthétique du boire éthiopien, norme reprise par l'industrie européenne et ses promoteurs en Éthiopie. Cette adaptation est donc liée à l'activité ethnographique des voyageurs-marchands de la fin du XIXe siècle comme Soleillet.

La collection de la mission Dakar-Djibouti comporte un wança en métal émaillé (fig. 12), dont la fiche descriptive précise qu'il a été donné par un négociant italien, Feliciano Zanotti, établi dans le bourg frontalier érythréen d'Om Häğär. II y est également souligné qu'« on en vend de semblables sur le marché d'Addis-Abeba", et qu'il s'agit d'un «objet de traite [...] d'usage général ${ }^{46}$ ». L'objet, de fabrication industrielle, reprend le décor habituel des wança - les filets parallèles en haut et en bas, ici dessinés en bleu sur le fond blanc - ainsi que leur forme évasée. II porte l'effigie de Menilek II, dessiné de profil, un motif commun aux premières pièces et aux premiers timbres éthiopiens frappés en 1884, alors dessinés et gravés par des artistes français pour être produits à Paris par l'Imprimerie nationale française (Sohier 2007, vol. I: 330-331). Comme sur les timbres, le médaillon ne comprend que des caractères éthiopiens. On comprend qu'il est indiqué en haut, dans une typographie amharique maladroite, "Sa majesté le roi des rois d'Éthiopie Menilek», et en bas la date éthiopienne de 1887 - 1894-1895 dans le calendrier grégorien. Autour du médaillon, en caractères latins, il est écrit - en bien plus gros - «INDUSTRIA ITALIANA». L'objet a donc 


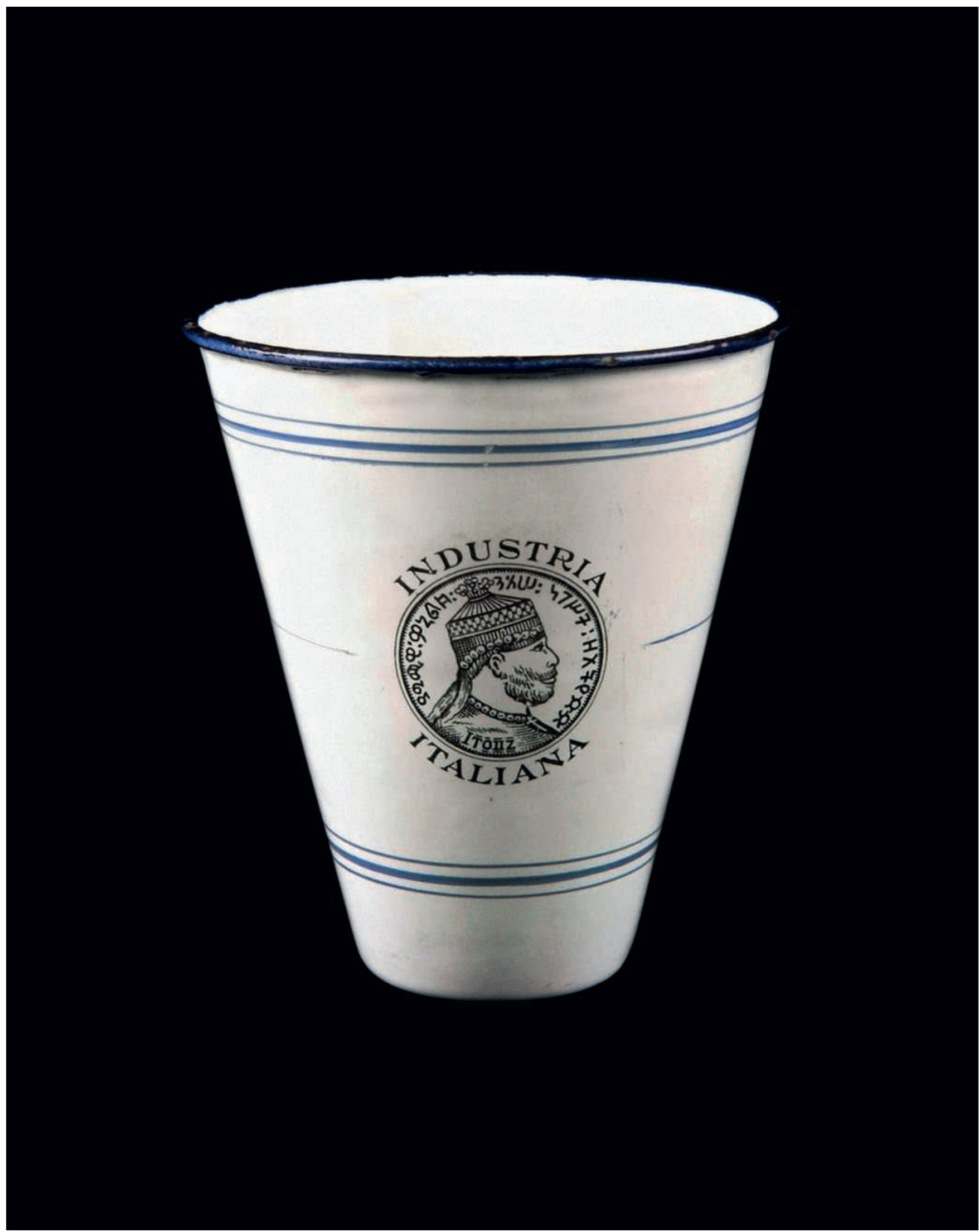


47. Les batailles d'Adwa et d'Ambalagē voient alors l'armée italienne défaite par les troupes éthiopiennes (Bahru Zewde 2002 [1991] : 76-80).

48. Voir par exemple les objets n ${ }^{\text {os }} 1847$ et 1848 (Menilek II), 1946 et 7984 (la reine Zäwditu), 1947 et 7342 (le régent Täfäri) ou 2805 (le lion de Judah seul).

49. Voir la fiche de l'objet n² 2806.

50. Voir la fiche de l'objet $n^{\circ} 1852$, qui mentionne explicitement une origine érythréenne («S.E.P.I. ASMARA-MASSAUA»).

51. Objet $n^{\circ} 1848$. été réalisé par une usine italienne - en Italie ou en Érythrée - dans le contexte tendu qui précède la première Guerre italo-éthiopienne 47 (1895-1896). Si I'on considère le potentiel de diffusion de tels objets en Éthiopie, ce message associant la figure du roi éthiopien à un produit manufacturé italien est de portée considérable. Par une boucle historique ironique, c'est Léon Chefneux, I'un des proches contacts de Soleillet auprès de Menilek II, qui a porté la commande royale de timbres à l'Imprimerie nationale française. La mission Dakar-Djibouti récupère donc en Érythrée, dans les années 1930, un objet dont le modèle a été généré au temps des premières collectes d'objets, dans les années 1880.

Élément isolé dans la collection du musée du quai Branly par son matériau et sa fabrication, ce wança connaît de nombreux équivalents dans la collection du musée de l'Institut des études éthiopiennes. On y trouve plusieurs gobelets à l'effigie de Menilek II, des modèles présentant le lion de Judah, avec ou sans les initiales de la reine des rois Zäwditu ou de son régent le ras Täfäri, qui attestent tous du rôle médiatico-politique de ces objets utilisés dans les banquets publics du début du $x x^{e}$ siècle $^{\mathbf{4 8}}$. II faut également signaler de nombreuses pièces sans décoration. Les quelques gobelets comportant la mention de leur origine renvoient à l'Italie ${ }^{\mathbf{4 9}}$, mais aussi - surtout? - à sa présence régionale en Érythrée ${ }^{50}$, ainsi qu'à la Tchécoslovaquie ${ }^{51}$. Ce dernier, nécessairement fabriqué après 1918, porte encore le sceau de Menilek II quelques années après la mort du souverain en 1913. La présence de ces objets de fabrication européenne vient souligner, dans le quotidien éthiopien, l'importance nouvelle du jeu international. Sans être elle-même colonisée, l'Éthiopie est entrée dans le temps colonial à la fin du XIX ${ }^{e}$ siècle. Alors que Menilek II lance son royaume dans une vague de conquêtes territoriales orientées vers le Sud-Ouest et le Sud-Est éthiopiens, I'Italie construit une colonie en Érythrée depuis 1890 avec son aval, intégrant une grande partie de la province chrétienne du Tegray. La France et le Royaume-Uni contrôlent, dès 1884, respectivement la baie de Tadjourah et les ports de Zeyla et de Berbera, sur la rive africaine du golfe d'Aden. Enfin, en accord avec Menilek II, la France fait construire entre 1897 et 1917 une ligne de chemin de fer entre son port de Djibouti et la capitale éthiopienne. La ville d'Addis-Abeba elle-même, nouvellement créée, comprend d'influentes communautés étrangères - arménienne, arabe, grecque et d'autres nations européennes. Cette présence renforcée de l'«étranger» (färänğ) tend à changer l'offre et les pratiques du boire dans leur dimension alimentaire et matérielle.

\section{Un nouvel ordre social pour le «boire éthiopien»}

La nouveauté du fait urbain entraîne l'apparition des premiers débits de boisson. Ceux-ci commercialisent le țäğğ et la țälla, alimentant la demande en récipients «normés». C'est à ce moment que l'eau-de-vie passe manifestement du statut de boisson des élites à celui d'alcool populaire. La production locale d'alcool distillé n'est pas attestée avant la fin du $\mathrm{XIX}$ siècle dans les sources historiques. II est probable que les eaux-devie consommées plus tôt à Gondär aient été des produits d'importation. Soleillet pourrait être le premier à avoir observé un alambic «de fabrication européenne ou indienne» dans un palais éthiopien, à la cour de Menilek II, dans le Šäwa, en 1882 (Soleillet 1886: 103). II signale toutefois que, «dans 
la plupart des maisons, ces appareils sont remplacés par des vases en terre et des cannes forées". La version locale de l'alambic d'importation correspond à ce qu'observe Griaule lors de son séjour à Gondär en 1932 - il faut néanmoins y ajouter un système de condensation dans un récipient métallique plongé dans l'eau froide ${ }^{52}$. Le schéma et les photographies que réalise l'ethnologue témoignent du réemploi d'une forme de céramique (mägäbärya) qui est ordinairement utilisée pour contenir la pâte servant à confectionner les galettes ${ }^{53}$ (enğära). L'alambic éthiopien est donc probablement l'imitation locale d'un alambic d'importation, réalisée avec des matériaux de récupération.

La chronique du règne de Menilek II associe eau-de-vie et țäğğ comme boissons servies aux plus hauts dignitaires du royaume lors du banquet royal ${ }^{\mathbf{5 4}}$. Le chroniqueur se lamente toutefois de la trop importante consommation d'araqē et de țäğğ à laquelle se livrent les ouvriers responsables de la construction de l'église royale d'Ențotțo Maryam (Guèbrè Sellassié 1930-1931, vol. I: 212). La boisson est lourdement condamnée quelques années plus tard par le Dr Mérab, qui en fait même l'origine d'un nouveau mal du xx siècle, l'«arékisme» (Mérab 1912: 122; 1929: 528). Ce témoin, inquiet de l'évolution en cours, compare très négativement d'araqē, que produiraient massivement les «Grecs» et les «Arméniens» d’Addis-Abeba - il est lui-même d'origine géorgienne -, aux boissons «hygiéniques» que constituent à ses yeux la țälla et le țäğğ (Mérab 1929: 543).

L'eau-de-vie est à l'origine d'une culture matérielle du boire spécifique. Les tourneurs de corne produisent de petits récipients, peut-être inspirés des tasses en porcelaine d'importation dont nous avons déjà parlé, désignés génériquement sous le terme mäläkkiya. Le terme renvoie à l'idée de mesure, le récipient fixant une norme commerciale de volume (Kane 1990, vol. I: 159). Dès 1885, un objet de ce type entre dans les collections françaises par le biais du don Soleillet ${ }^{55}$ (fig. 10). Les calices (sewa, fig. 11), verres à pied fabriqués en corne également présents dans les collections, peuvent aussi avoir servi à boire l'eau-de-vie. Mérab signale également que celle qu'on produit en Éthiopie est conditionnée dans des bouteilles de verre importées (țermus) [Mérab 1929: 529]. La démocratisation du goût pour les alcools distillés s'accompagne en effet d'une conquête progressive du marché éthiopien par l'industrie du verre, comme le montre, dans les réserves du musée du quai Branly, une enseigne rapportée d'Addis-Abeba par la mission Dakar-Djibouti et vraisemblablement produite pour une araqē bēt («maison» de l'eau-de-vie, débit de boisson). On y observe des récipients transparents et même un tonneau de conception très européenne (fig. 14).

L'augmentation de l'offre en alcools importés - vin, alcools distillés puis produits localement modifie considérablement la pratique du boire des élites sociales des premières décennies du xx siècle (ibid.: 509 ; Rouaud 2009: 137-140). Là encore, tout n'est pas qu'une affaire de goût: il s'agit aussi d'objets. Lorsque le ras Haylu du Goğğam se fait représenter présidant un banquet auquel assistent Griaule et son comparse, Maurice Larget, un ensemble de bouteilles bouchées et étiquetées sont disposées sur sa table. L'œuvre, conservée au musée du quai Branly, a été réalisée par un peintre de cour, «Wuddye d'Addiet ${ }^{56}$ », lors de la mission de 1928-1929.
52. Fonds Marcel Griaule/ Papiers/Grands fichiers/ Fichier Éthiopie 1931-1937. Catégorie "Alimentation"; sous-catégorie

"Alimentation". Réf. fiche: fr/up10/mae/lesc/fmg_pap _gf_aet3137_002_003. Voir également les photographies fonds "Mission Dakar-Djibouti " /Série L/Pellicule 74, clichés 5 à 8.

53. Fonds Marcel Griaule/ Papiers/Grands fichiers/ Fichier Éthiopie 1931-1937. Catégorie «Ustensiles ». Réf. fiche: fr/up10/mae/lesc/fmg_ pap_gf_aet3137_130_042.

54. Guèbrè Sellassié 1930-1931, vol. I: 226. La description du banquet de Menilek II dans le Zekra nägär de l'archiviste et ancien ministre Mahtämä Śelässē comporte également une allusion à l'araqēe, mais celui-ci est servi à des officiers moins prestigieux (Mahtämä Śelässē Wäldä Masqal 1949: 47).

55. $N^{\circ}$ d'inv. MQB 71.1885.22.61.

56. Addēt est alors la capitale du ras.

\section{double page suivante}

fig. 13

Enseigne de débit de boisson, araqē bēt (don mission Dakar-Djibouti, 1931). Musée du quai Branly $n^{\circ} 71.1931 .74 .3445$.

(C) musée du quai Branly. 


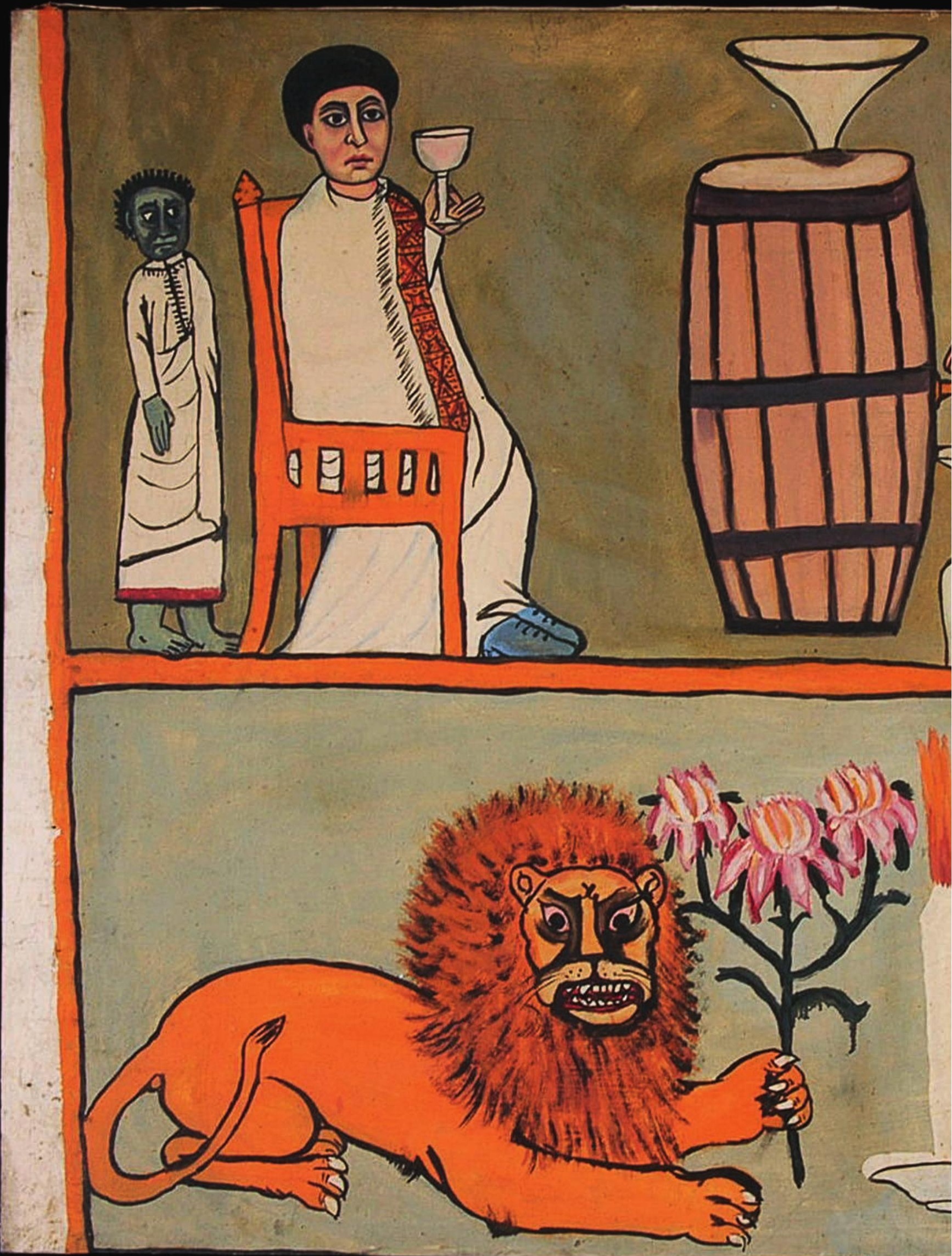




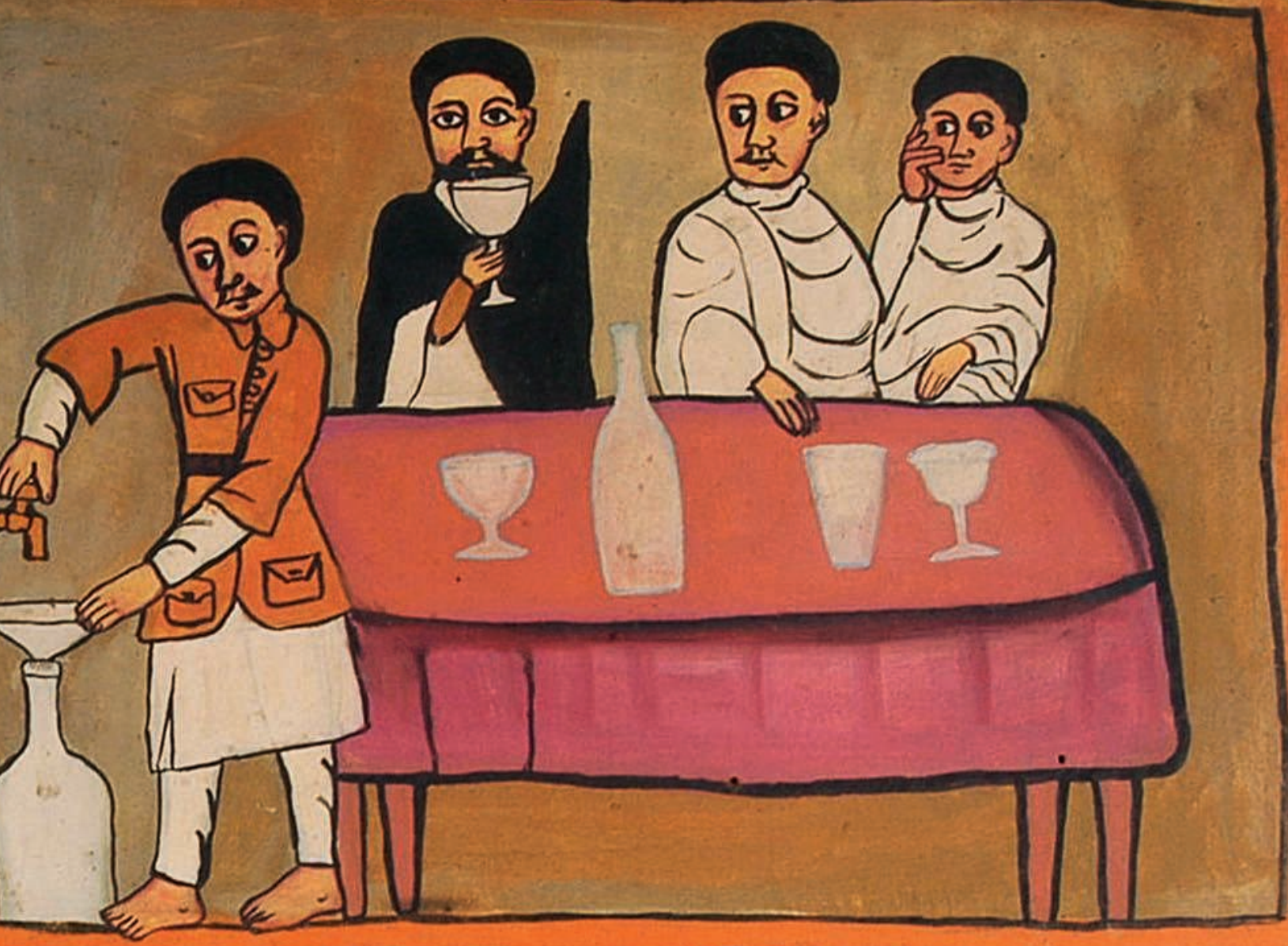




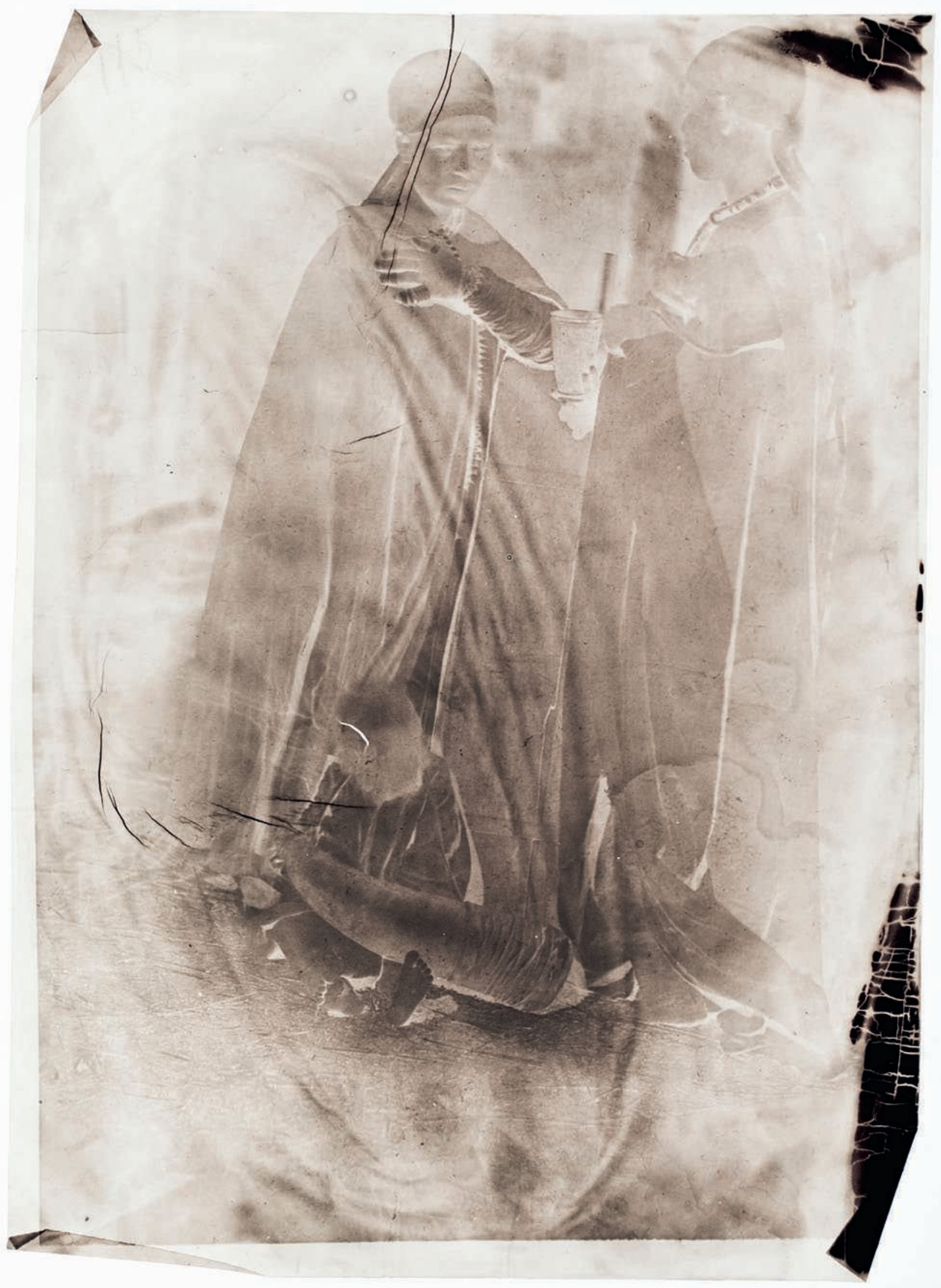


C'est au palais de ce même ras que Griaule a également observé les expériences d'un échanson produisant du țäğğ pétillant, bouché et étiqueté (Griaule 1934). Sur ce tableau particulièrement révélateur du nouvel ordre social de la boisson, le ras Haylu boit, dans un calice doré mais translucide ${ }^{\mathbf{5 7}}$, un liquide rouge qui pourrait être du vin. C'est aussi le cas des deux Français à sa gauche et à sa droite. La «foule» du banquet boit plus ordinairement du țäğğ dans des berellē. Sur les tables des deux étrangers, un calice est posé à côté d'un wança métallique blanc portant les deux filets de couleur foncée. Ce dernier comporte en son centre une initiale, la lettre éthiopienne ha (3), comme Haylu. Le ras récupère ici le média de communication employé par les souverains éthiopiens.

En intégrant à une composition classique, une scène de banquet, des éléments nouveaux comme un wança de fabrication industrielle, des bouteilles de vin et même des färänğ européens, l'artiste et son commanditaire témoignent de la volonté d'associer des aspects de la modernité matérielle occidentale aux pratiques séculaires de la table éthiopienne. Cet affichage est autant destiné aux publics éthiopien qu'étrangers, les dignitaires étant alors de plus en plus amenés à accueillir des représentants de nations d'outre-mer. De ce processus mêlant logiques internes et externes à l'Éthiopie et à sa société peuvent attester à la fois les sources historiques textuelles et les collections ethnographiques.

Les collections ethnographiques éthiopiennes sont une partie importante du corpus de sources qui documentent l'histoire de cette région. Avec leurs biais propres, qu'il s'agit d'identifier, elles constituent une séquence chronologique d'environ un siècle et demi pour le cas présent. L'exemple de la culture matérielle du boire témoigne de fortes évolutions, rapides, concernant l'une des catégories de mobilier les plus socialement valorisées. Loin d'une représentation statique de l'univers matériel domestique, elles révèlent, dans la continuité des sources historiques textuelles plus anciennes, une succession de modes, d'innovations et de banalisations. Elles permettent également de souligner le caractère «connecté» de l'histoire de la culture matérielle, et ce avec une certaine profondeur historique. Une «connexion» qui s'accélère au xIx ${ }^{e}$ siècle, à travers l'activité des voyageurs, puis des collectionneurs, l'intérêt ethnographique pour l'Éthiopie se développant en même temps que le marché éthiopien s'ouvre aux appétits européens. Les collections éclairent certains aspects méconnus de la circulation des objets, de l'évolution du goût pour ceux-ci ainsi que des savoir-faire inhérents à leur fabrication au sein de l'ensemble régional éthiopien, en particulier avant les conquêtes de la fin du $x x^{e}$ siècle. L'étude permet en effet de mettre en lumière de fortes convergences entre différentes sociétés sur le plan de la culture matérielle. Existe-t-il une culture du boire «oromo» distincte d'une culture du boire «amhara»? Dès le xIx ${ }^{e}$ siècle, rien n'est moins sûr. t.guindeuil@gmail.com
57. Une fausse coupe en or offerte à Raymond Poincaré a été au cœur d'une polémique diplomatico-littéraire au retour de la première mission Griaule en Éthiopie. II pourrai s'agir d'une représentation de celle-ci (voir Griaule 1991 [1934] : 155 ; Armandy 1930 : 198-199; ainsi que les articles et lettres ouvertes publiés à ce sujet dans le journal

Comœdia paru le 17 octobre et le 14 novembre 1930). Je remercie Éric Jolly d'avoir attiré mon attention sur le lien possible entre ce tableau et cette affaire, qui marque le lancement de la mission Dakar-Djibouti.

\section{ci-contre}

fig. 14

Deux femmes éthiopiennes conversent, l'une avec un gobelet wança en bois dans la main (entre 1885 et 1888). Épreuve négative. Photo Jules Borelli. @ ) musée du quai Branly.

\section{remerciements}

Cet article a été réalisé dans le cadre d'une bourse de recherche du musée du quai Branly (2012-2013). II est issu d'une présentation discutée au sein du séminaire du département de la Recherche et de l'Enseignement du musée du quai Branly, organisé par Julien Clément, Jessica De Largy Healy et Anne-Christine Taylor. Une première version du texte avait alors été débattue avec Éloi Ficquet, Éric Jolly et Dominique Juhé-Beaulaton, ainsi qu'avec les membres du département et les autres boursiers. Mes remerciements vont à ce chaleureux auditoire qui a joué un grand rôle dans la formulation finale de l'article. Je remercie également le musée du quai Branly pour avoir rendu possible un séjour à Addis-Abeba, au musée de l'Institut des études éthiopiennes, permettant ainsi de confronter les collections parisiennes aux collections conservées en Éthiopie. 


\section{Abbadie, Antoine d'}

1881 Dictionnaire de la langue amariñña. Paris, F. Veweg. [“Actes de la Société philologique", t. X].

1890 Géographie de l'Éthiopie: ce que j'ai entendu, faisant suite à ce que j'ai vu. Paris, Gustave Mesnil.

\section{Abbebe Kifleyesus}

2002 "Muslims and meals: The social and symbolic function of food in changing socio-economic environments ", Africa LXXII (2): 245-276.

2011 "In vino veritas: "Wine" and moonshine and the language of drinking and drunkenness in Debre Bïrhan ", Annales d'Éthiopie XXVI: 207-246.

\section{Abbink, Jon}

1997 «Competing practices of drinking and power: Alcoholic "hegemonism" in Southern Ethiopia", Northeast African Studies IV (3), nouvelle série: $7-22$

2005 "Drinks ", in Siegbert Uhlig (dir.), Encyclopaedia Aethiopica, vol. V. Wiesbaden, Harrassowitz Verlag: 198-200.

\section{Adjemian, Boris}

2011 Immigrants arméniens, représentations de l'étranger et construction du national en Éthiopie ( $x I X^{\theta}-x x^{\theta}$ siècle): socio-histoire d'un espace interstitiel de sociabilités, 2 vol. Thèse de doctorat d'histoire, EHESS-Università degli Studi di Napoli «L'Orientale».

\section{Ahmad, Abdussamad H.}

1988 "Hunting in Gojjam: The case of Matakal 1901-1932 ", in Tadesse Beyenne (dir.), Proceedings of the Eighth International Conference of Ethiopian Studies, University of Addis Ababa, 1984, vol. I. AddisAbeba et Francfort-sur-le-Main, Institute of Ethiopian StudiesGoëthe Universität: 237-244.

\section{Álvares, Francisco}

1961 The Prester John of the Indies. A True Relation of the Lands of the Prester John, 2 vol., Charles Fraser Beckingham et George Wynn Brereton Huntingford (trad. et éd.). New York, The Hakluyt SocietyCambridge University Press.

\section{Ancel, Stéphane}

2011 "Le coût de la mort dans les communautés chrétiennes en Éthiopie", Afriques. Débats, méthodes et terrains d'histoire 3 ; http://afriques.revues.org/925.

\section{Armandy, André}

1930 La Désagréable Partie de campagne. Incursion en Abyssinie. Paris, Alphonse Lemerre.

\section{Baeteman, Joseph}

1929 Dictionnaire amarignafrançais, suivi d'un vocabulaire français-amarigna. Diré Dawa, Imprimerie Saint-Lazare.

\section{Bahru Zewde}

2002 [1991] A History of Modern Ethiopia, 1855-1991. Addis-Abeba, Oxford et Athens, Addis Ababa University Press-James CurreyOhio University Press.

\section{Beckingham, Charles}

Fraser et Huntingford, George Wynn Brereton (trad. et éd.)

1954 Some Records of Ethiopia, 1593-1646. Londres, The Hakluyt Society.

\section{Beke, Charles T.}

1844 "Abyssinia - Being a continuation of routes in that country ", Journal of the Royal Geographical Society XIV: 1-76.

\section{Biaso, Elisabeth}

2004 Prunk und Pracht am Hofe Menileks. Alfred llgs Äthiopien um 1900/Majesty and magnificience at the Court of Menilek. Alfred IIg's Ethiopia around 1900. Zurich, Verlag Neue Zürcher Zeitung/Völkerkundemuseum der Universität Zürich.

\section{Boardman, Sheila}

1999 «The agricultural foundation of the Aksumite Empire, Ethiopia: An interim report ", in Marijke van der Veen (dir.), The Exploitation of Plant Resources in Ancient Africa. New York, Kluwer AcademicsPlenum Publishers: 137-148.

\section{Bonacci, Giulia}

2011 Exodus! L'histoire du retour des Rastafariens en Éthiopie. Paris, L'Harmattan.

\section{Borelli, Jules}

1890 Éthiopie méridionale. Journal de mon voyage aux pays amhara, oromo et sidama. Septembre 1885 à novembre 1888. Paris, Librairies-Imprimeries réunies.

\section{Broc, Numa}

1988 Dictionnaire illustré des explorateurs et grands voyageurs français $d u \times x x^{e}$ siècle, vol. I, Afrique. Paris, Éditions du C.T.H.S.

\section{Campbell, Ian}

2011 "The bïrillē man of Harer: The contribution of Arthur Rimbaud to the evolution of a uniquely Ethiopian drinking-vessel ", Annales d'Éthiopie XXVI: 179-205.

\section{Cheesman, Robert E.}

1936 Lake Tana \& Blue Nile: an Abyssinian Quest. Londres, MacMillan \& Co.

\section{Cohen, Marcel}

1912 Rapport sur une mission linguistique en Abyssinie (1910-1911). Paris, Imprimerie nationale.

\section{Combes, Edmond et Tamisier, Maurice}

1838 Voyage en Abyssinie, dans le pays des Galla, de Choa et d'Ifat, précédé d'une excursion dans l'Arabie-Heureuse, 5 vol.

Paris, E. Dessessart.

\section{Dubois, Colette}

1989 «Miel et sucre en Afrique orientale, 1830-1870: complémentarité ou concurrence?", The International Journal of African Historical Studies XXII (3) : 453-472.

\section{Duchesne-Fournet, Jean}

1909 Mission en Éthiopie (19011903), 2 vol. Paris, Masson et Cie.

\section{Eshete Tadesse}

1959 «Preparation of täğ among the Amhara of Šäwa ", University College of Addis Ababa Ethnological Society Bulletin I (9) : 101-108 [revue rééditée en 2002 en un volume unique, voir Pankhurst (A.) 2002].

\section{Éthiopie d'aujourd'hui. La terre et les hommes}

1975 Paris, Laboratoire d'ethnologie/ Museum national d'histoire naturelle/ musée de l'Homme.

\section{Ferret, Adolphe et} Galinier, Joseph Germain

1847-1848 Voyage en Abyssinie dans les provinces du Tigré, du Samen et de l'Amhara, 3 vol. Paris, Paulin.

\section{Griaule, Marcel}

1934 "D'un mode aberrant de conservation de l'hydromel au Godjam », Journal de la Société des africanistes IV (2) : 279-284.

1941 "Moules et tour à travailler la corne (Abyssinie) ", Journal de la Société des africanistes XI : 201-207.

1991 [1934] Les Flambeurs d'hommes. Paris, Berg International.

\section{Guèbrè Sellassié}

1930-1931 Chronique du règne de Ménélik II, roi des rois d'Éthiopie, 3 vol., Tèsfa Sellassié (trad.), Maurice de Coppet (éd.). Paris, Maisonneuve Frères.

\section{Guidi, Ignazio}

(trad. et éd.)

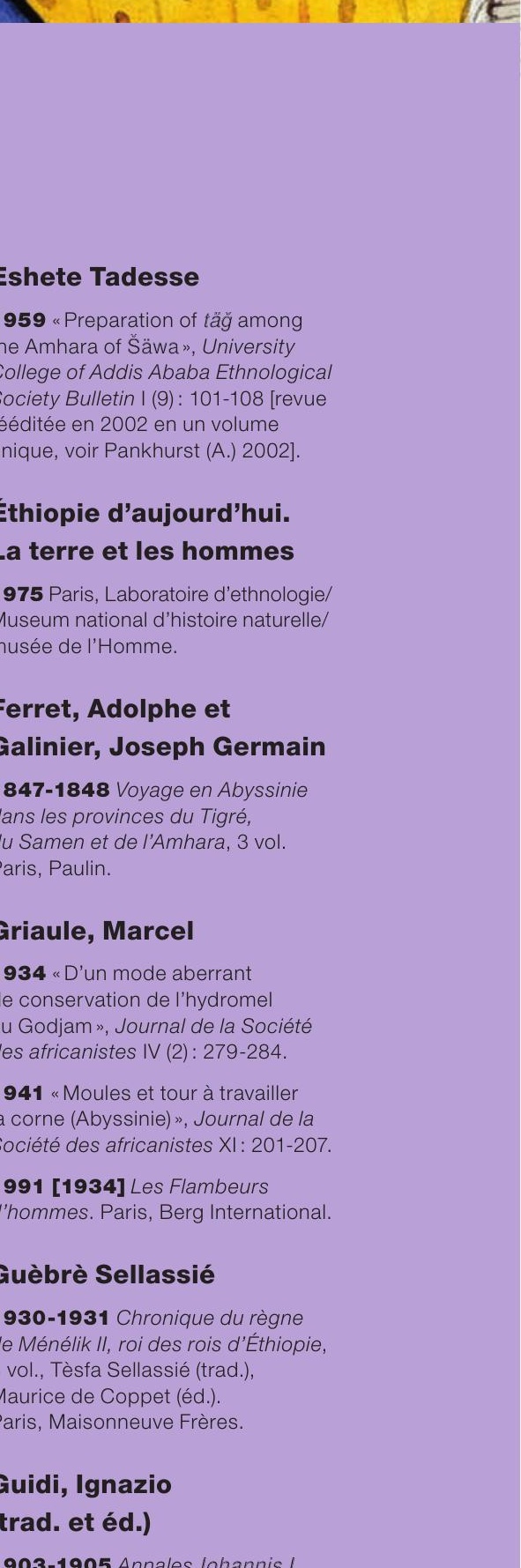

1903-1905 Annales Iohannis I lyāsu l et Bakāffā, 4 vol. Leipzig, Otto Harrassowitz ["Corpus Scriptorum Christianorum Orientalium, Scriptores Aethiopici, series altera» t. V].

1910-1912 Annales Regum lyāsull et lyo'as, 2 vol. Leipzig, Otto Harrassowitz ["Corpus Scriptorum Christianorum Orientalium, Scriptores Aethiopici, series altera» t. VI]. 


\section{Guindeuil, Thomas}

2010 " "Pour l'âne, le miel n'a pas de goût". Miel et société dans l'histoire du royaume d'Éthiopie ", Journal des africanistes LXXX (1-2) : 283-306.

2012 Alimentation, cuisine et ordre social dans le royaume d'Éthiopie $\left(x I^{e}-X I x^{e}\right.$ siècle). Thèse de doctorat d'histoire, université Paris I-Panthéon Sorbonne.

\section{Hecht, Elisabeth-Dorothea}

1969 The Pottery Collection.

Addis-Abeba, The Museum of the Institute of Ethiopian StudiesHaile Selassie I University.

\section{Imbert-Vier, Simon}

2011 Tracer des frontières à Djibouti: des territoires et des hommes aux $x x^{\ominus}$ et $x x^{\ominus}$ siècles. Paris, Karthala.

\section{Isenberg, Charles William}

1841 Dictionary of the Amharic Language. Londres, The Church Missionary Society.

\section{Johnston, Charles}

1844 Travels in Southern Abyssinia, through the Country of Adal to the Kingdom of Shoa, 2 vol. Londres,

J. Madden \& Co.

\section{Kane, Thomas Leiper}

1990 Amharic-English Dictionary,

2 vol. Wiesbaden, Otto Harrassowitz.

\section{Kropp, Manfred (éd.)}

1988 "The Sør'atä Gøbr: A mirror view of daily life at the Ethiopian royal court in the Middle Ages ", Northeast African Studies X (2-3) : 51-87.

\section{Leiris, Michel}

1996 [1934] "L'Afrique fantôme " in Michel Leiris (Jean Jamin et Jacques Mercier, éd.), Miroir de l'Afrique. Paris, Gallimard: 101-868.

\section{Mahtämä Śelassē Wäldä Mäsqäl}

1949 Hhઢ. h'C [Zekra nägär]. Addis-Abeba, Artistik Matämiyä Bēt.
McCann, James C.

2009 Stirring the Pot. A History of African Cuisine. Athens, Ohio University Press.

\section{Ménard, Diane (trad.)}

2004 Le Voyage aux Indes de Nicolò de' Conti (1414-1439).

Paris, Chandeigne.

\section{Mérab, Étienne}

1912 Médecins et médecine en Éthiopie. Paris, Vigot Frères.

1929 Impressions d'Éthiopie (L'Abyssinie sous Ménélik II), t. III. Institutions civiles (mariage, justice etc.), vie morale et matérielle des Abyssins. Paris, E. Leroux.

\section{Mission scientifique du Bourg de Bozas}

1906 De la mer Rouge

à l'Atlantique à travers l'Afrique tropicale (octobre 1900-mai 1903).

Paris, F.R. de Rudeval.

\section{Munro-Hay, Stuart}

1991 Aksum. An African Civilisation of Late Antiquity. Édimbourg, Edinburgh University Press.

\section{Páez, Pedro}

2008 História da Etiópia, Isabe Boavida, Hervé Pennec et Manuel João Ramos (éd.). Lisbonne, Assírio \& Alvim.

\section{Pankhurst, Alula}

2002 "The Ethnological Society Bulletin. An "age of cultural renaissance" ? ", in Alula Pankhurst (éd.), Addis Ababa University College Ethnological Society Bulletin, 1953-1961. Addis-Abeba, Addis Ababa University : xiv-xxiii.

\section{Pankhurst, Richard}

1985 The History of Famin and Epidemics in Ethiopia prior to the Twentieth Century. Addis-Abeba, Relief and Rehabilitation Commission.

2003 "Armenians", in Siegber Uhlig (dir.), Encyclopaedia Aethiopica, vol. I. Wiesbaden, Harrassowitz Verlag: 344-347.

\section{Parkyns, Mansfield}

1868 Life in Abyssinia. Being Notes Collected during Three Year's Residence and Travels in that Country. Londres, John Murray.

\section{Poncet, Jacques-Charles}

2010 Relation de mon voyage

d'Éthiopie, 1698-1701,

Éric Poix (éd.). Besançon,

Éditions la Lanterne magique.

\section{Rochet d'Héricourt,} Charles-François-Xavier

1841 Voyage sur la côte orientale de la mer Rouge, dans le pays d'Adel et le royaume de Choa.

Paris, Arthus Bertrand.

\section{Rouaud, Alain}

1999 «Tracé biographique», in Alain Rouaud (dir.), Les orientalistes sont des aventuriers. Guirlande offerte à Joseph Tubiana par ses élèves et ses amis. Saint-Maur-des-Fossés, Sépia: 287-289.

2009 " Boire et trop boire en Éthiopie chrétienne: mots, objets, pratiques ", Acta orientalia belgica XXII («René Lebrun in honorem.

Vin, bière et ivresse dans

es civilisations orientales.

Entre plaisir et interdit»): 127-144.

\section{Salt, Henry}

1814 A Voyage to Abyssinia and Travels into the Interior of that Country, Executed under the Orders of the British Government in the Years 1809 and 1910. Londres, F.C. and J. Rivington/W. Bulmer and Co.

\section{Saurat, Anne, Ahmad Zekaria et Aklilu Yilma}

1989 Musée de l'Institut des études éthiopiennes (université d'Addis-Abeba). Guide-catalogue. Addis-Abeba, Institut des études éthiopiennes.

\section{Sohier, Estelle}

2007 Politiques de l'image et pouvoir royal en Éthiopie de Menilek I à Haylä Sellasé (1880-1936), 2 vol. Thèse de doctorat d'histoire, université Paris Panthéon Sorbonne/Università degli Studi di Napoli «L'Orientale».

\section{Soleillet, Paul}

1886 Voyages en Éthiopie: janvier 1882-octobre 1884 Rouen, Espérance Cagnard.

\section{Tegegne Yetesha Work}

1958 "Tälla", University College of Addis Ababa Ethnological Society Bulletin I (8) : 77-82 [revue rééditée en 2002 en un volume unique, voir Pankhurst (A.) 2002].

\section{Valentia, George A.}

1809 Voyages and Travels to India, Ceylon, the Red Sea, Abyssinia and Egypt in the Years 1802, 1803, 1804, 1805, and 1806, 3 vol. Londres, William Miller. 